\title{
FROM BINARY GROUPS TO TERMINAL RINGS
}

\author{
Stuart D. Scott $1, *$ \\ ${ }^{1}$ Department of Mathematics, University of Auckland, Auckland, New Zealand \\ Communicated by Erchard Aichinger \\ Original Research Paper \\ Received: Oct 3, 2019 . Accepted: Jul 5, 2021 \\ First published online: October 8, 2021 \\ ๑) 2021 The Author(s)
}

\begin{abstract}
Binary groups are a meaningful step up from non-associative rings and nearrings. It makes sense to study them in terms of their nearrings of zero-fixing polynomial maps. As this involves algebras of a more specialized nature these are looked into in sections three and four. One of the main theorems of this paper occurs in section five where it is shown that a binary group $V$ is a $P_{0}(V)$ ring module if, and only if, it is a rather restricted form of non-associative ring. Properties of these non-associative rings (called terminal rings) are investigated in sections six and seven. The finite case is of special interest since here terminal rings of odd order really are quite restricted. Sections eight to thirteen are taken up with the study of terminal rings of order $p^{n}$ ( $p$ an odd prime and $n \geq 1$ an integer $\leq 7$ ).
\end{abstract}

\section{KEYWORDS}

nearring, binary group, anti-associative ring, anti-commutative ring, inverse ring, terminal ring

MATHEMATICS SUBJECT CLASSIFICATION (2020)

Primary 08A05; Secondary 17D99, 16Y30

\section{INTRODUCING BINARY GROUPS}

One of the more fundamental notions of abstract algebra is that of a group. Groups tend to arise in nearly all branches of this subject. In this paper we are involved in the study of groups with an additional operation. In these investigations certain types of non-associative rings will arise. One in particular will be studied. It will be these non-associative rings that allow us to determine when the group structure is a ring module relative to the nearring of zero-fixing polynomial maps.

Traditionally in the study of groups the binary operation has been multiplication (ie. that denoted by juxtaposition). As is the case with nearrings this paper uses additive notation. Here the notation used is not meant to imply the groups involved have to be commutative. In accordance with our additive convention the identity (ie. additive identity) of a group $V$ is denoted by 0 . Also if $S$ is a subset of $V, S \backslash\{0\}$ is written as $S^{*}$ and $\langle S\rangle$ is taken as the subgroup of $V$ generated by $S$.

The reason additive convention for groups is useful is that it streamlines binary group (now defined) studies. A binary group is a group with a further binary operation for which multiplicative notation is used. Thus, if $V$ is such a group and $\gamma$ its further operation, then $(u, v) \gamma(u \in V$ and $v \in V)$ is denoted by $u v$.

\footnotetext{
${ }^{*}$ Corresponding author. E-mail: stuartdonaldscott@gmail.com
} 
With a slight exception in the case of certain nearrings the binary groups we study will be assumed to be zero-symmetric. Thus in general a binary group $V$ will be considered to have the property that $0 u=u 0=0$ for all $u$ in $V$. Sometimes $V$ may have an identity (a particular element denoted by 1 and such that $u 1=1 u=u$ for all $u$ in $V$ ). When this is the case the 1 of $V$ is unique. We indicate $V$ has such an element by calling it a binary group with 1 . Following on fairly naturally from the definition of a binary group comes the notion of a binary subgroup. $U$ is such a subobject of the binary group $V$ when it is an additive subgroup of $V$ with the property that $u_{1} u_{2}$ is in $U$ for all $u_{i}, i=1,2$, in $U$.

Looking into matters to do with subsets of binary groups is perhaps the next step in their investigation. Here, for two non-empty subsets $S_{1}$ and $S_{2}$ of such a group $V, S_{1}+S_{2}$ and $S_{1} S_{2}$ are respectively defined to be $\left\{s_{1}+s_{2}: s_{1} \in S_{1}, s_{2} \in S_{2}\right\}$ and $\left\{s_{1} s_{2}: s_{1} \in S_{1}, s_{2} \in S_{2}\right\}$. As the addition and multiplication of $V$ cannot be expected to be commutative, it is not necessarily the case that $S_{1}+S_{2}=S_{2}+S_{1}$ or that $S_{1} S_{2}=S_{2} S_{1}$. Furthermore, for $S_{3}$ another non-empty subset of $V$, it is not necessarily the case that $\left(S_{1} S_{2}\right) S_{3}=S_{1}\left(S_{2} S_{3}\right)$ although addition of non-empty subsets is certainly associative. It appears that particular subsets of $V$ are of greater interest. These are the right (left) $V$-subsets. Here a non-empty subset $S$ of $V$ is a right (left) $V$-subset when $S V$ (when $V S$ ) is contained in $S$. When $S$ is also an additive subgroup of $V$ it is called a right (left) $V$-subgroup. In the case of it being both a right and left $V$-subset ( $V$-subgroup) it is simply called a $V$-subset ( $V$-subgroup).

Two things about binary groups deserving of attention are a natural relationship between them and what this relationship throws up (ie. ideals). These matters are approached here from an $\Omega$-group (that is an expanded group) perspective. $\Omega$-groups have been about for a long time (see [2]) and it is assumed that in that direction the reader is familiar with the basics. $\Omega$-group definitions present us with homomorphisms between binary groups. Also isomorphism theorems are available. The ideals of the binary group $V$ are just the kernels of homomorphisms on $V$. Alternatively an ideal of $V$ is a normal subgroup $U$ of $V$ (ie. of $(V,+))$ for which $\left(v_{1}+u_{1}\right)\left(v_{2}+u_{2}\right)-v_{1} v_{2}$ is in $U$ for all $v_{i}, i=1,2$, in $V$ and $u_{i}, i=1,2$, in $U$. Such an ideal $A$ of $V$ supplies us with the binary group $V / A$ for which $\left(w_{1}+A\right)+\left(w_{2}+A\right)=w_{1}+w_{2}+A$ and $\left(w_{1}+A\right)\left(w_{2}+A\right)=w_{1} w_{2}+A\left(w_{i}, i=1,2\right.$, in $\left.V\right)$. In the above definition of ideals using elements of $V$, we do not need to assume the subgroup $U$ is normal when $V$ is binary with 1 . This is because with $v_{2}=1$ and $u_{2}=0$ we see $v_{1}+u_{1}-v_{1}$ is in $U$. Also, because zero-symmetry is being assumed, we have:

PROPOSITION 1.1. If $V$ is a binary group, then an ideal $U$ of $V$ is a $V$-subgroup of $U$.

Proof. In the above definition of ideals using elements of $V$, we see on taking $u_{1}=0$ and $v_{2}=0$, that $v_{1} u_{2}$ is in $U$. On taking $v_{1}=0$ and $u_{2}=0$ it follows that $u_{1} v_{2}$ is in $U$. Proposition 1.1 is proved.

From $\Omega$-group considerations it follows that any sum of ideals of a binary group is an ideal. A direct sum of ideals of such a group is defined in the standard manner. We also have that sums of two subobjects where only one is an ideal tend to fall into line. Here, if $U$ is taken respectively to be a binary subgroup, right (left) $V$-subgroup or just a $V$-subgroup of the binary group $V$, then the same holds for $U+A(A$ an ideal of $V)$.

If $V$ is a binary group and $k$ an integer $\geq 1$ and $v_{i}, i=1, \ldots, k$, elements of $V$ then, when associativity is present, the product $v_{1} v_{2} \ldots v_{k}$ has a precise meaning. This may not be the case for general $V$ and overcoming the problem involved here means defining product sets. This is done inductively. For a single element $a$ of $V$ the product set $\lfloor a\rfloor$ of $V$ is taken as $\{a\}$. If for some integer $n \geq 1$ all product sets $\left\lfloor a_{1}, \ldots, a_{m}\right\rfloor\left(1 \leq m \leq n\right.$ and $a_{i}, i=1, \ldots, n$, in $\left.V\right)$ are defined, then the product set $\left\lfloor v_{1}, v_{2}, \ldots, v_{n+1}\right\rfloor\left(v_{i}, i=1, \ldots, n+1\right.$, in $\left.V\right)$ is taken as $\cup_{m=1}^{n}\left(\left\lfloor v_{1}, \ldots, v_{m}\right\rfloor .\left\lfloor v_{m+1}, \ldots, v_{n+1}\right\rfloor\right)$. This definition will be of help latter. Developments of section three and four will make quite real use of it.

In this paper a number of different algebraic structures arise. The more general are binary groups and the more specific are terminal rings (see section five). Making the transition from one extreme to the other is, in part, what this paper is about. It is investigations into polynomial maps on binary groups that find us within a terminal ring environment. The question of when polynomial maps distribute over an ideal is central to this. 
Nearrings of polynomial maps are a powerful tool in binary group (in $\Omega$-group) investigations. As has been indicated above they lead to rather meaningful research possibilities. The next section will introduce the nearrings $P(V)$ and $P_{0}(V)$ ( $V$ a binary group) of polynomial maps.

\section{NEARRINGS $P(V)$ AND $P_{0}(V)$ ( $V$ A BINARY GROUP)}

If maps of a group into itself are being considered, then there often arises another structure. In such situations nearrings often come into play. First attempts to investigate them go back a long way (see [3]) and this paper assumes the reader has some knowledge of both nearrings and nearring groups. A basic text on them is [4]. Here, unlike [4], we will be using the left distributive law (ie. $\alpha(\beta+\gamma)=\alpha \beta+\alpha \gamma)$ rather than the right. This of course means functions are written on the right.

An $N$-group $V$ ( $N$ zero-symmetric) is called tame (see [8]) if $V$ is a unitary $N$-group with the property that all $N$-subgroups are submodules (ie. $N$-ideals). This is characterized by requiring that for each $v$ and $w$ in $V$ and $\alpha$ in $N$, we can find a $\beta$ in $N$ such that $(v+w) \alpha-v \alpha=w \beta$. In the situation where $\beta$ can be found independently of $w, V$ is called a compatible $N$-group. Although compatible $N$-groups are considerably more restricted than tame ones, they are still very plentiful (see $\S 5$ of [10]). When a nearring $N$ has a faithful tame (compatible) $N$-group ( $V$ say) it is said to be tame (compatible) or more precisely tame (compatible) on $V$.

As is common in nearring theory the symbols $M(V)\left(M_{0}(V)\right)$ are used to denote the nearring of all (all zero fixing) functions of a group $V$ into itself. $M_{0}(V)$ is a zero symmetric nearring while $M(V)$ is only zero symmetric when $V=\{0\} . M(V)$ plays a somewhat further role when $V$ is a binary group. Here it is not only a nearring under pointwise addition and composition but a binary group under a rather natural multipication. This multiplication is that given by taking the product $\alpha . \beta$ ( $\alpha$ and $\beta$ in $M(V))$ to be the function of $M(V)$ taking $v$ in $V$ to $(v \alpha)(v \beta)$. It is the binary subgroup $P(V)$ of $M(V)$ generated by $\{1\} \cup C(C$ the subnearring of constant maps of $M(V))$ that will be of interest and in order to study it we inductively define certain subsets $\Gamma_{n}(V), n=1,2, \ldots$. Here $\Gamma_{1}(V)=\{1\} \cup C$ and for $n \geq 2, \Gamma_{n}(V)$ is taken as

$$
\Gamma_{n-1}(V) \cup\left\{\alpha . \beta, \alpha-\beta: \alpha, \beta \in \Gamma_{n-1}(V)\right\} .
$$

The notation $\Gamma_{n}(V), n=1,2, \ldots$, has been introduced because it occurs again in section four. Clearly $\Gamma_{1}(V) \subseteq \Gamma_{2}(V) \subseteq \ldots$ and $P(V)=\cup_{n=1}^{\infty} \Gamma_{n}(V)$. Certainly $P(V)$ is a binary subgroup of $M(V)$ but something more is true.

In the more general context of $V$ being an $\Omega$-group the $\Omega$-subgroup $P(V)$ of $M(V)$ is readily defined (see Section 7.118 onwards of [4]). Moreover, it is a subnearring of $M(V)$ called the nearring of polynomial maps of $V$ into $V$. This nearring is of considerable use in the study of $V$. Perhaps even more useful is the zero-symmetric part $P_{0}(V)$ of $P(V)$. Either of these $N\left(P(V)\right.$ or $\left.P_{0}(V)\right)$ have $V$ as a faithful $N$-group. In the case of the $P_{0}(V)$-group $V$ an important fact emerges. This is that $P_{0}(V)$ is compatible on $V$. Thus, in accordance with what was set out above, $P_{0}(V)$-subgroups of $V$ are submodules. However, more is true.

It is of considerable help in investigating an $\Omega$-group $V$ to view it as a $P_{0}(V)$-group. Taken on the surface the fact that $V$ is a compatible $P_{0}(V)$-group does not seem to say a great deal. A reason for this is that first impressions are that the $P_{0}(V)$-subgroups of $V$ may not be related in any way much to $\Omega$-group structure. The fact that they are actually the ideals (see Section 7.123 of [4]) means much more becomes possible. On restricting to binary groups Section 7.123 of [4] becomes:

THEOREM 2.1. Suppose $V$ is a binary group. $U$ is a $P_{0}(V)$-subgroup of $V$ if, and only if, it is an ideal.

It would seem that due to Theorem 2.1 any serious attempt to understand binary groups must mean viewing them as $P_{0}(V)$-groups. An important aspect of this paper is that of investigating the $U$ (as in Theorem 2.1) which are $P_{0}(V)$ ring modules. 


\section{ANTI-ASSOCIATIVE RINGS}

In this paper the term ring will in general mean non-associative ring. On the whole only when $P_{0}(V)$-ring modules are being considered or the word associative is used is the associative law operating.

Binary groups can satisfy laws other than zero-symmetry. These more restricted algebras can be of very real interest. One such class is that of non-associative rings. These are just additively abelian binary groups satisfying both distributive laws. The past has seen considerable attention paid to particular subclasses. Here interest has focused on such things as Lie rings, Jordan rings and alternative rings (see [5]). In the study of them the additional laws give them their unique character. This is certainly the case for associative rings resting so much as they do on associativity. Associativity can seem fundamental but when studying binary groups another law sometimes tends to take its place. A subclass of the class of non-associative rings is that of anti-associative rings. Here, a non-associative ring $V$ is an anti-associative ring if $u(v w)=-(u v) w$ for all $u, v$ and $w$ in $V$. The purpose of the present section is to prove a result about the subset $\left\lfloor v_{1}, v_{2}, \ldots, v_{k}\right\rfloor(k \geq 3$ an integer and $v_{i}, i=1, \ldots, k$, in $V$ ) of the anti-associative ring $V$. To do this we introduce certain specialized products of a binary group.

Suppose $V$ is a binary group and $u_{1}, u_{2}, u_{3}, \ldots$ a sequence of elements of $V$. We let $\xi_{l}\left(u_{1}\right)(l$ stands for left) be $u_{1}$ and with $n \geq 1$ an integer and $\xi_{l}\left(u_{1}, \ldots, u_{n}\right)$ defined, $\xi_{l}\left(u_{1}, \ldots, u_{n+1}\right)$ is taken as $\xi_{l}\left(u_{1}, \ldots, u_{n}\right) u_{n+1}$. Thus the sequence, $\xi_{l}\left(u_{1}\right), \xi_{l}\left(u_{1}, u_{2}\right), \xi_{l}\left(u_{1}, u_{2}, u_{4}\right), \xi_{l}\left(u_{1}, u_{2}, u_{3}, u_{4}\right), \ldots$ is just the sequence, $u_{1}, u_{1} u_{2},\left(u_{1} u_{2}\right) u_{3},\left(\left(u_{1} u_{2}\right) u_{3}\right) u_{4}, \ldots$ of elements of $V$. A right handed version of $\xi_{l}$ will also be of use. Here $\xi_{r}\left(u_{1}\right)=u_{1}$ and for $n \geq 2$ an integer $\xi_{r}\left(u_{1}, \ldots, u_{n}\right)$ is defined inductively as $u_{n} \xi_{r}\left(u_{1}, \ldots, u_{n-1}\right)$. Thus the sequence $\xi_{r}\left(u_{1}\right), \xi_{r}\left(u_{1}, u_{2}\right), \xi_{r}\left(u_{1}, u_{2}, u_{3}\right), \xi_{r}\left(u_{1}, u_{2}, u_{3}, u_{4}\right), \ldots$ is just the sequence, $u_{1}, u_{2} u_{1}, u_{3}\left(u_{2} u_{1}\right), u_{4}\left(u_{3}\left(u_{2} u_{1}\right)\right), \ldots$ of elements of $V$.

If $V$ is an anti-associatuve ring, $k \geq 1$ an integer and $v_{1}, \ldots, v_{k}$, elements of $V$, then much can be said about the subset $\left\lfloor v_{1}, \ldots, v_{k}\right\rfloor$ of $V$. Clearly for $k=1$ or $2,\left\lfloor v_{1}, \ldots, v_{k}\right\rfloor$ is respectively $v_{1}$ and $v_{1} v_{2}$ but for $k \geq 3$ things are also simple enough. The theorem this section proves is:

THEOREM 3.1. Suppose $V$ is an anti-associative ring and $k \geq 3$ an integer. If $v_{1}, \ldots, v_{k}$, are elements of $V$, then $\left\lfloor v_{1}, \ldots, v_{k}\right\rfloor$ has the form $\{d,-d\}$ where $d$ is an element of $V$.

Proof. It will first be shown that $\left\lfloor v_{1}, \ldots, v_{k}\right\rfloor \subseteq\{d,-d\}$ where $d$ is an element of $V$. To do this suppose $n \geq 1$ is an integer minimal such that there exists $u_{1}, \ldots, u_{n}$ in $V$ with $\left\lfloor u_{1}, \ldots, u_{n}\right\rfloor$ not contained in such a subset of $V$. If this assumption leads to a contradiction the first part of the proof will be complete.

Because for $n=1$ or $2,\left\lfloor u_{1}, \ldots, u_{n}\right\rfloor$ is a single element of $V, n \geq 3$. Now $c$ in $\left\lfloor u_{1}, \ldots, u_{n}\right\rfloor$ is of the form $c_{1} c_{2}$ with $c_{1}$ in $\left\lfloor u_{1}, \ldots, u_{a}\right\rfloor$ and $c_{2}$ in $\left\lfloor u_{a+1}, \ldots, u_{n}\right\rfloor(a$ an integer $\geq 1$ and $<n)$. The minimality of $n$ forces $\left\lfloor u_{1}, \ldots, u_{a}\right\rfloor \subseteq\left\{d_{1},-d_{1}\right\}$ and $\left\lfloor u_{a+1}, \ldots, u_{n}\right\rfloor \subseteq\left\{d_{2},-d_{2}\right\}$ where $d_{1}$ and $d_{2}$ are in $V$. Thus $c$ is in $\left\{ \pm \xi_{l}\left(u_{1}, \ldots, u_{a}\right) \cdot \xi_{r}\left(u_{n}, u_{n-1}, \ldots, u_{a+1}\right)\right\}$. However, for $a+1=n$

$$
\xi_{l}\left(u_{1}, \ldots, u_{a}\right) \cdot \xi_{r}\left(u_{n}, \ldots, u_{a+1}\right)=\xi_{l}\left(u_{1}, \ldots, u_{a}\right) \cdot u_{n}=\xi_{l}\left(u_{1}, \ldots, u_{n}\right)
$$

and for $a+1<n$

$$
\xi_{l}\left(u_{1}, \ldots, u_{a}\right) \cdot \xi_{r}\left(u_{n}, \ldots, u_{a+1}\right)=\xi_{l}\left(u_{1}, \ldots, u_{a}\right)\left(u_{a+1} \cdot \xi_{r}\left(u_{n}, \ldots, u_{a+2}\right)\right),
$$

where the right hand side is $-\left[\xi_{l}\left(u_{1}, \ldots, u_{a+1}\right) \cdot \xi_{r}\left(u_{n}, \ldots, u_{a+2}\right)\right]$. Continuing in this manner we see $\left\{\xi_{l}\left(u_{1}, \ldots, u_{a}\right) \cdot \xi_{r}\left(u_{n}, \ldots, u_{a+1}\right)\right\}$ is necessarily contained in $\left\{ \pm \xi_{l}\left(u_{1}, \ldots, u_{n}\right)\right\}$. Thus $c$ is contained in $\left\{ \pm \xi_{l}\left(u_{1}, \ldots, u_{n}\right)\right\}$ and, by contradiction, the first part of the proof is complete.

To complete the proof we must show that if $k \geq 3$ and $g$ is in $\left\lfloor v_{1}, \ldots, v_{k}\right\rfloor$, then $-g$ is in $\left\lfloor v_{1}, \ldots, v_{k}\right\rfloor$. Here $g$ is of the form $g_{1} g_{2}$ with $g_{1}$ in $\left\lfloor v_{1}, \ldots, v_{b}\right\rfloor$ and $g_{2}$ in $\left\lfloor v_{b+1}, \ldots, v_{k}\right\rfloor(b$ an integer $\geq 1$ and $<k)$. Two cases arise. These are where $b=1$ or $b \geq 2$. In the first we have $k-b \geq 2$ and this situation is in many ways like the second. Therefore we deal with the second case only. Here $g_{1}$ is of the form $g_{3} g_{4}$ with $g_{3}$ in $\left\lfloor v_{1}, \ldots, v_{e}\right\rfloor$ and $g_{4}$ in $\left\lfloor v_{e+1}, \ldots, v_{b}\right\rfloor(e \geq 1$ an integer $<b)$. Thus $g=\left(g_{3} g_{4}\right) g_{2}$ and $g_{3}\left(g_{4} g_{2}\right)$ is also in $\left\lfloor v_{1}, \ldots, v_{k}\right\rfloor$. Since $g_{3}\left(g_{4} g_{2}\right)=-g$ the theorem is proved. 


\section{POWER ASSOCIATIVITY AND INVERSE RINGS}

The last section dealt with anti-associative rings. It was proved that in such a non-associative ring $V$ a product set $\left\lfloor v_{1}, \ldots, v_{k}\right\rfloor\left(k \geq 1\right.$ an integer and $v_{i}, i=1, \ldots, k$, in $\left.V\right)$ has at most two elements. In the proof of this the anti-associative law was used and also the distributive laws. When it comes to associativity rather than anti-associativity a well known much more general result is possible. Here, in a binary group with a multiplicative associative law (called an associative binary group), product sets consist of a single element. Also such a condition on product sets implies the binary group is associative. However, moving away from all product sets being singletons, to requiring this for some of a select nature means much more general binary groups can occur. A binary group $V$ will be called power associative if any product set $\left\lfloor v_{1}, \ldots, v_{k}\right\rfloor$ in which all the $v_{i}$ are equal (equal to $v$ say) is a singleton. In this case the natural notation $v^{k}$ will be used to denote this element of $V$.

Power associative binary groups are defined. Clearly an anti-associative ring $V$ will be power associative (see Theorem 3.1) if all product sets $\left\lfloor v_{1}, v_{2}, \ldots, v_{k}\right\rfloor(k \geq 2)$ of $V$ with the $v_{i}$ equal have an element of additive order $\leq 2$. There is a reasonably natural (quite strong) condition on $V$ that ensures this. A non-associative ring $V$ which is anti-commutative (ie. satisfies $u v=-v u$ for all $u$ and $v$ in $V$ ) will be called an anti-commutative ring. If an anti-associative ring is also anti-commutative it will be called an inverse ring.

PROPOSITION 4.1. Suppose $V$ is an anti-commutative ring and $\left\lfloor v_{1}, \ldots, v_{k}\right\rfloor$ a product set of $V$ where all the $v_{i}$ are equal. If $k \geq 2$, then all elements of $\left\lfloor v_{1}, \ldots, v_{k}\right\rfloor$ have additive order $\leq 2$.

Proof. There exists $v$ in $V$ such that $v_{i}=v$ for $i=1, \ldots, k$. When $k=2,\left\lfloor v_{1}, \ldots, v_{k}\right\rfloor$ consists of the single element $v v$ and since, by anti-commutativity $v v=-v v$, Proposition 4.1 holds. Suppose it has been shown that for all integers $a \geq 2$ and $<k,\left\lfloor v_{1}, \ldots, v_{a}\right\rfloor$ consists of elements of additive order $\leq 2$. If $k \geq 3$, then an element $c$ of $\left\lfloor v_{1}, \ldots, v_{k}\right\rfloor$ is of the form $c_{1} c_{2}$ where $c_{1}$ is in $\left\lfloor v_{1}, \ldots, v_{b}\right\rfloor$ and $c_{2}$ in $\left\lfloor v_{b+1}, \ldots, v_{k}\right\rfloor\left(b\right.$ an integer $\geq 1$ and $<k$ ) so that either $c_{1}$ or $c_{2}$ is in $\left\lfloor v_{1}, \ldots, v_{e}\right\rfloor$ ( $e$ an integer $\geq 2$ and $<k$ ). Thus either $c_{1}$ or $c_{2}$ has additive order $\leq 2$. Since distributive laws hold $|c| \leq 2$ and Proposition 4.1 has been inductively proved.

COROLLARY 4.2. An inverse ring is power associative.

Proof. By Proposition 4.1, this follows from Theorem 3.1.

For a binary group $V$ elements of $P(V)$ can, in a sense, be thought of as defined inductively in terms of the $\Gamma_{n}(V), n=1,2, \ldots$. Here we have $P(V)=\cup_{n=1}^{\infty} \Gamma_{n}(V)$ where $\Gamma_{1}(V)$ is specified and $\Gamma_{n}(V)$ $(n \geq 2)$ is given in terms of $\Gamma_{n-1}(V)$. While very useful, this definition of $P(V)$ does not help much with visualizing $P(V)$ 's elements. Indeed, in general, it is hard to know what elements of $P(V)$ look like. However, when $V$ is an inverse ring things are different. Here, elements of $P(V)$ can be specified in terms of those of type (6.1), (8.1) or (10.1) (to be defined). Type (6.1), (8.1) and (10.1) maps also exist in power associative binary groups. Although they tell us little in general about elements of $P(V)$ ( $V$ a power associative binary group) it is in this wider context they will be defined.

Let $V$ be a power associative binary group. Type (6.1) elements of $P(V)$ are taken as the constant maps (ie. those mapping all of $V$ to a single element of $V$ ). Type (8.1) elements of $P(V)$ are taken as those maps mapping $v$ in $V$ to $v^{n}$ (to $-v^{n}$ ) where $n \geq 1$ is an integer. Type (10.1) elements of $P(V)$ are taken as maps taking $v$ in $V$ to $u . v^{n}(u \in V$ and $n \geq 1$ an integer). Type (6.1), (8.1) and (10.1) elements of $P(V)$ are called elementary and $E(V)$ is defined as $\{\alpha \in P(V): \alpha$ of type (6.1), (8.1) or (10.1) $\}$ while the zero symmetric part $E(V) \backslash\{\alpha \in P(V): \alpha$ of type (6.1) $\}$ of $E(V)$ will be denoted by $E_{0}(V)$.

Let $V$ be a binary group. $P(V)$ is not only a nearring under substitution and pointwise addition but there is another naturally defined multiplication on $P(V)$ extending that of $V$ and making $P(V)$ into a binary group. In the case of $V$ being an inverse ring this multiplication makes $P(V)$ into an inverse ring. In this situation the product $E(V) . E(V)$ of the subset $E(V)$ with itself is $\subseteq E(V)$. We also have $-E(V)=\{-\alpha: \alpha \in E(V)\}$ is $E(V)$. This second statement is easily proved. All that really needs to be observed is that $-\left(u \cdot v^{n}\right)(u \in V$ and $n \geq 1$ an integer $)$ is $(-u) . v^{n}$. The first statement's

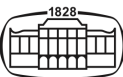


proof is omitted. It is easy enough to check out. For example part of the proof consists of showing $\left(u_{1} v^{n_{1}}\right) \cdot\left(u_{2} \cdot v^{n_{2}}\right)\left(u_{i}, i=1,2\right.$. in $V$ and $n_{i} \geq 1$ integers $)$ is equal to

$$
-\left(u_{1}\left(v^{n_{1}}\left(u_{2} v^{n_{2}}\right)\right)\right)=u_{1}\left(\left(v^{n_{1}} u_{2}\right) v^{n_{2}}\right)=-\left(u_{1}\left(\left(u_{2} v^{n_{1}}\right) v^{n_{2}}\right)\right)=u_{1}\left(u_{2} \cdot v^{n_{1}+n_{2}}\right)
$$

and this is just $-\left(\left(u_{1} u_{2}\right) \cdot v^{n_{1}+n_{2}}\right)=\left(-u_{1} u_{2}\right) v^{n_{1}+n_{2}}$.

A proposition formalizes what has been indicated.

PROPOSITION 4.3. If $V$ is an inverse ring, then $-E(V)=E(V)$ and $E(V) \cdot E(V)$ is contained in $E(V)$.

The time has come to see just what elements of $P(V)$ ( $V$ an inverse ring) look like.

THEOREM 4.4. An element $\alpha$ of $P(V)$ ( $V$ an inverse ring) is of the form $\sum_{i=1}^{n} \alpha_{i}$ where the $\alpha_{i}$ are in $E(V)$.

Proof. Suppose there exist elements of $P(V)$ not of the desired form. Furthermore, suppose the integer $n$ is chosen minimal such that $\Gamma_{n}(V)$ contains such an element ( $\beta$ say). Since all elements of $\Gamma_{1}(V)$ are in $E(V)$ it follows that $n \geq 2$. The nature of $\beta$ and $n$ imply $\beta=\beta_{1}-\beta_{2}$ or $\beta=\beta_{1} \beta_{2}$ where $\beta_{1}=\gamma_{1}+\cdots+\gamma_{n_{1}}\left(n_{1} \geq 1\right.$ an integer and $\gamma_{i}, i=1, \ldots, n_{1}$, in $\left.E(V)\right)$ and $\beta_{2}=\lambda_{1}+\ldots, \lambda_{n_{2}}\left(n_{2} \geq 1\right.$ an integer and $\lambda_{i}, i=1, \ldots, n_{2}$, in $E(V)$ ). However, as

$$
\beta_{1}-\beta_{2}=\gamma_{1}+\cdots+\gamma_{n_{1}}-\lambda_{n_{2}}-\cdots-\lambda_{1} \quad \text { and } \quad \beta_{1} \beta_{2}=\sum_{i=1}^{n_{1}} \sum_{j=1}^{n_{2}} \gamma_{i} \lambda_{j},
$$

Proposition 4.3 supplies us with the contradiction that $\beta_{1}-\beta_{2}$ and $\beta_{1} \beta_{2}$ are a finite sum of elements of $E(V)$. Theorem 4.4 has been proved.

COROLLARY 4.5. An element $\alpha$ of $P_{0}(V)$ ( $V$ an inverse ring) is of the form $\sum_{i=1}^{n} \alpha_{i}$ where the $\alpha_{i}$ are in $E_{0}(V)$.

Proof. By Theorem 4.4, $\alpha=\sum_{i=1}^{n} \alpha_{i}$ where the $\alpha_{i}$ are in $E(V)$. If all the $\alpha_{i}$ are of type (8.1) or (10.1), then $\alpha$ has the expression indicated. If none of the $\alpha_{i}$ are of type (8.1) or (10.1), then $\alpha$, being constant, is zero. In this situation $\alpha=1+(-1)$ where 1 and -1 are of type (8.1). If just some of the $\alpha_{i}$ are of type (8.1) or type (10.1), then $\alpha=\beta_{1}+\cdots+\beta_{n_{1}}+\gamma_{1}+\cdots+\gamma_{n_{2}}\left(n_{i}, i=1,2\right.$ integers $\geq 1$ and the $\beta_{j}$ of type (6.1) and the $\gamma_{j}$ of type (8.1) or (10.1)). From the fact that $0 \alpha=0$ we conclude that $\beta_{1}+\cdots+\beta_{n_{1}}=0$ and $\alpha$ has the expression indicated. Corollary 4.5 has been proved.

\section{TERMINAL RINGS AND $P_{0}(V)$ RING SUBMODULES}

Up until now there has been increasing specialization in the structures being considered. We have moved from binary groups to non-associative rings and then to anti-associative and anticommutative rings. These have been followed (see the last section) by the introduction of inverse rings. We now take a step further in this direction with the introduction of terminal rings (to be defined). The name would seem appropriate as they allow one of the main goals of this paper to be reached. The goal here is the determination of when a binary group $V$ is a $P_{0}(V)$-ring module.

An inverse ring has been defined as a non-associative ring which is both anti-associative and anti-commutative. Such non-associative rings are considerably restricted. For example, in an inverse ring $V$, elements of $P(V)$ have a rather basic form (see Theorem 4.4). However, if the goal is to specify when $V$ is a $P_{0}(V)$ ring module a further important condition is necessary. We shall call an inverse ring $V$ a terminal ring if $v^{2} u+u^{2} v=0$ for all $u$ and $v$ in $V$.

Anti-associativity and anti-commutativity are, in some sense, quite natural conditions. By comparison the law $v^{2} u+u^{2} v=0$ seems strange. It might be expected to have a very limiting effect. This is the case. It implies powers of elements of $V$ are quite strongly restricted.

PROPOSITION 5.1. Any element $v$ of an terminal ring must be such that $v^{4}=v^{5}=v^{6}=\ldots$.

Proof. By Proposition 4.1 and Corollary 4.2, it follows that when the $u$ of $v^{2} u+u^{2} v=0$ is replaced by $v^{2}$ we obtain $v^{4}=v^{5}$. Thus, $v^{4} v=v^{5} v\left(v^{5}=v^{6}\right)$ etc. Proposition 5.1 is proved. 
If $V$ is a binary group and $U$ a $P_{0}(V)$-subgroup of $V$, then it is a $V$-subgroup of $V$ (see Proposition 1.1 and Theorem 2.1) and, of course, a binary subgroup. It would appear that in general not much can be said about such binary subgroups. However, when $U$ is a $P_{0}(V)$ ring submodule, things are different. This fact is largely a consequence of a proposition about how elements of $U$ and $V$ interact.

PROPOSITION 5.2. If $V$ is a binary group and $U$ a $P_{0}(V)$ ring submodule of $V$, then $\left(u_{1}+u_{2}\right) w=$ $u_{1} w+u_{2} w, w\left(u_{1}+u_{2}\right)=w u_{1}+w u_{2}$ and $\left(u_{1} w\right) u_{2}=-\left(u_{2} w\right) u_{1}$, for all $u_{i}, i=1,2$, in $U$ and $w$ in $V$.

Proof. $(U,+)$ is an abelian group and an element $\alpha$ of $P_{0}(V)$ has the property that $\left(u_{1}+u_{2}\right) \alpha=$ $u_{1} \alpha+u_{2} \alpha$. Taking $\alpha$ as the map of $P_{0}(V)$ taking $v$ of $V$ to $v w$ we see $\left(u_{1}+u_{2}\right) w=u_{1} w+u_{2} w$. When $\alpha$ is taken as the map mapping $v$ in $V$ to $w v$ the fact that $w\left(u_{1}+u_{2}\right)=w u_{1}+w u_{2}$ is obtained. With $\alpha$ the map taking $v$ in $V$ to $(v w) v$ we see $\left(\left(u_{1}+u_{2}\right) w\right)\left(u_{1}+u_{2}\right)=\left(u_{1} w\right) u_{1}+\left(u_{2} w\right) u_{2}$. Because for all $u_{i}, i=1,2$, in $U$ and $w$ in $V,\left(u_{1}+u_{2}\right) w=u_{1} w+u_{2} w, w\left(u_{1}+u_{2}\right)=w u_{1}+w u_{2}$ and the $u_{i} w$ are in $U$, the left hand side of this expression is

$$
\left(u_{1} w+u_{2} w\right)\left(u_{1}+u_{2}\right)=\left(u_{1} w\right) u_{1}+\left(u_{2} w\right) u_{1}+\left(u_{1} w\right) u_{2}+\left(u_{2} w\right) u_{2}
$$

so that $\left(u_{2} w\right) u_{1}+\left(u_{1} w\right) u_{2}=0$. This means the third equation of Proposition 5.2's statement holds. Proposition 5.2 is proved.

Proposition 5.2 allows the proof that $U$ must be a terminal ring.

THEOREM 5.3. Suppose $U$ is a $P_{0}(V)$ ring submodule of the binary group $V$. When $U$ is viewed as a binary group it is a terminal ring.

Proof. $(U,+)$ is abelian and, by Proposition 5.2, the binary group $U$ satisfies both distributive laws. To show it is a terminal ring we must prove it is anti-commutative, anti-associative (the inverse ring conditions) and that $v^{2} u+u^{2} v=0$ for all $u$ and $v$ in $V$.

To prove $U$ is an inverse ring note, because the map taking $x$ in $V$ to $x x$ is in $P_{0}(V),\left(u_{1}+\right.$ $\left.u_{2}\right)\left(u_{1}+u_{2}\right)=u_{1} u_{1}+u_{2} u_{2}$ for all $u_{i}, i=1,2$, in $U$. Here, distributive laws imply $\left(u_{1}+u_{2}\right)\left(u_{1}+u_{2}\right)=$ $u_{1} u_{1}+u_{1} u_{2}+u_{2} u_{1}+u_{2} u_{2}$ so that $u_{1} u_{2}+u_{2} u_{1}=0$. With $u_{3}$ also in $U$, it follows from Proposition 5.2 that $\left(u_{1} u_{2}\right) u_{3}=-\left(u_{3} u_{2}\right) u_{1}$ where, by anti-commutativity, $-\left(u_{3} u_{2}\right) u_{1}=\left(u_{2} u_{3}\right) u_{1}=-u_{1}\left(u_{2} u_{3}\right)$. Thus $U$ is an inverse ring and is power associative.

To prove $v^{2} u+u^{2} v=0$ for all $u$ and $v$ in $V$ we use the distributivity over $U$ of the maps taking $x$ in $V$ to $x^{2}$ and $x$ in $V$ to $x^{3}$. This implies

$$
(v+u)^{3}=v^{3}+u^{3}=(v+u)^{2}(v+u)=\left(v^{2}+u^{2}\right)(v+u)=v^{3}+v^{2} u+u^{2} v+u^{3}
$$

from which the terminal nature of $U$ follows. Theorem 5.3 is proved.

When the $U$ of Theorem 5.3 can be taken as $V$ we have an 'if and only if' result.

THEOREM 5.4. The binary group $V$ is a $P_{0}(V)$ ring module if, and only if, it is a terminal ring.

Proof. By Theorem 5.3, when $V$ is a $P_{0}(V)$ ring module it has to be a terminal ring. Suppose $V$ is a terminal ring. Certainly it is an inverse ring. $(V,+)$ is abelian and Corollary 4.5 ensures that an $\alpha$ of $P_{0}(V)$ is of the form $\sum_{i=1}^{n} \alpha_{i}$ where the elements $\alpha_{i}$ of $P_{0}(V)$ are of type (8.1) or type (10.1). Clearly, if it is shown that for any given $i$ in $\{1, \ldots, n\}$ and $v_{j}, j=1,2$, in $V,\left(v_{1}+v_{2}\right) \alpha_{i}=v_{1} \alpha_{i}+v_{2} \alpha_{i}$, then Theorem 5.4 will be proved. Each $\alpha_{i}$ is a map taking $v$ in $V$ to $v^{r}$ (to $-v^{r}$ ) or to $u . v^{r}(u \in V)$ where $r$ is an integer $\geq 1$ and $\leq 4$ (see Proposition 5.1). It follows readily from this that $\alpha_{i}$ will have the desired property if, for such an $r,\left(v_{1}+v_{2}\right)^{r}=v_{1}^{r}+v_{2}^{r}$. For $r=1$, this is trivial. For $r=2$, we have $\left(v_{1}+v_{2}\right)^{2}=v_{1}^{2}+v_{1} v_{2}+v_{2} v_{1}+v_{2}^{2}=v_{1}^{2}+v_{2}^{2}$ by anti-commutativity. For $r=3$, it follows that

$$
\left(v_{1}+v_{2}\right)^{3}=\left(v_{1}+v_{2}\right)^{2}\left(v_{1}+v_{2}\right)=\left(v_{1}^{2}+v_{2}^{2}\right)\left(v_{1}+v_{2}\right)=v_{1}^{3}+v_{1}^{2} v_{2}+v_{2}^{2} v_{1}+v_{2}^{3}
$$

where the terminal nature of $V$ ensures the right hand side is $v_{1}^{3}+v_{2}^{3}$. For $r=4$, it follows that

$$
\left(v_{1}+v_{2}\right)^{4}=\left(v_{1}+v_{2}\right)^{2}\left(v_{1}+v_{2}\right)^{2}=\left(v_{1}^{2}+v_{2}^{2}\right)^{2}=v_{1}^{4}+v_{1}^{2} v_{2}^{2}+v_{2}^{2} v_{1}^{2}+v_{2}^{4}
$$

where the anti-commutativity of $V$ ensures the right hand side is $v_{1}^{4}+v_{2}^{4}$. The theorem is proved.

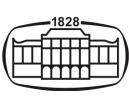




\section{DECOMPOSITION OF TERMINAL RINGS}

There is a certain amount of overlap between the class of rings (associative) and anti-associative rings. Here, any ring in which all elements are of additive order $\leq 2$ is anti-associative. However, rings in which all elements have order $\leq 2$ are not necessarily anti-commutative and certainly may not be terminal.

When it comes to studying terminal rings a subclass of rings stands out. Boolean rings are easily shown to be terminal rings and can, when an identity is present, be the dominant feature.

PROPOSITION 6.1. A binary group $V$ with 1 is a $P_{0}(V)$ ring module if, and only if, $V$ is a Boolean ring.

Proof. Since a Boolean ring is a terminal ring it follows from Theorem 5.4 that, when $V$ is Boolean, it is a $P_{0}(V)$ ring module. On the other hand when $V$ is a $P_{0}(V)$ ring module Theorem 5.4 implies $V$ is terminal. If in the expression $v^{2} u+u^{2} v=0(u$ and $v$ in $V)$ we take $u=1$, then it follows that $v^{2}+v=0$. But now Proposition 4.1 implies $v^{2}=v$ for all $v$ in $V$ and all $v$ have additive order $\leq 2$. This last fact implies the associative law holds. Thus $V$ is a Boolean ring and Proposition 6.1 is completely proved.

At least one special case of Proposition 6.1 is already known. Section 7.128 of [4] deals with $V$ being a ring with identity.

Proposition 6.1 seems to say a $P_{0}(V)$ ring module $V$ is rare. However, an alternative view is that the existence of an identity is a restrictive requirement. This viewpoint tends to be reinforced by more general binary group studies. In fact Boolean rings often only enter into things as part of the picture. We now set about making this plain.

If $V$ is a terminal ring, then we let $\mathcal{E}(V)$ be the set of all $e$ in $V$ such that $e^{2}=e$. We let $\mathcal{N}(V)$ be all $v$ in $V$, such that $v^{n}=0$ for some integer $n \geq 1$. $\mathcal{E}(V)$ and $\mathcal{N}(V)$ supply a decomposition of the terminal ring $V$.

THEOREM 6.2. If $V$ is a terminal ring, then $\mathcal{E}(V)$ and $\mathcal{N}(V)$ are ideals of $V$. Furthermore, $V=\mathcal{E}(V) \oplus$ $\mathcal{N}(V)$ and $v^{4}=0$ for all $v$ in $\mathcal{N}(V)$.

Proof. In a non-associative ring $R$ an $R$-subgroup $A$ is an ideal. This follows because $(A,+)$ is a normal $\operatorname{subgroup~of~}(R,+)$ and for $r_{i}, i=1,2$, in $R$ and $a_{i}$ in $A,\left(r_{1}+a_{1}\right)\left(r_{2}+a_{2}\right)-r_{1} r_{2}=r_{1} a_{2}+r_{2} a_{1}+a_{1} a_{2}$. Thus, to prove the first statement of the theorem it is sufficient to show $\mathcal{E}(V)$ and $\mathcal{N}(V)$ are $V$-subgroups of $V$.

If $e_{i}, i=1,2$, are in $\mathcal{E}(V)$, then as $e_{2}^{2}=e_{2}, e_{2}$ has order $\leq 2$ and by anti-commutativity

$$
\left(e_{1}-e_{2}\right)^{2}=\left(e_{1}+e_{2}\right)^{2}=e_{1}^{2}+e_{1} e_{2}+e_{2} e_{1}+e_{2}^{2}=e_{1}+e_{2}=e_{1}-e_{2} .
$$

Thus $\mathcal{E}(V)$ is a subgroup of $V$. For $e$ in $\mathcal{E}(V)$ and $u$ in $V$ we have $e^{2} \cdot u+u^{2} \cdot e=0$. Here $e^{2} \cdot u=e u$ while anti-associativity and anti-commutativity imply

$$
u^{2} e=(u u)(e e)=-u(u(e e))=u((u e) e)=-u((e u) e)=u(e(u e))=-(u e)(u e)
$$

so that the right hand side is $-(e u)^{2}$ and $(e u)^{2}=e u$. This means $\mathcal{E}(V)$ is a right $V$-subgroup of $V$ and by anti-commutativity it is a $V$-subgroup.

To prove $\mathcal{N}(V)$ is a subgroup of $V$ it is first indicated how $v^{4}=0$ for all $v$ in $\mathcal{N}(V)$. Because $v$ is in $\mathcal{N}(V), v^{n}=0$ for some integer $n \geq 1$. If $n=1,2$ or 3 reasonably elementary considerations imply $v^{4}=0$. If $n \geq 4$ then, by Proposition 5.1, $v^{4}=0$. We now note that if $u_{i}, i=1,2$, are in $\mathcal{N}(V)$, then $\left(u_{1}-u_{2}\right)^{2}=u_{1}^{2}+\left(-u_{2}\right)^{2}=u_{1}^{2}+u_{2}^{2}$ so that $\left(u_{1}-u_{2}\right)^{4}=\left(u_{1}^{2}+u_{2}^{2}\right)^{2}=u_{1}^{4}+u_{2}^{4}=0$. Thus $\mathcal{N}(V)$ is a subgroup of $V$. If $a$ is in $V$, then anti-commutativity and anti-associativity imply $\left(a u_{1}\right)^{2}=-a^{2} \cdot u_{1}^{2}=\left(-a^{2}\right) \cdot u_{1}^{2}$. As $u_{1}^{2}$ is in $\mathcal{N}(V)$ this in turn yields $\left(a u_{1}\right)^{4}=\left(\left(-a^{2}\right) \cdot u_{1}^{2}\right)^{2}=\left[-\left(-a^{2}\right)^{2}\right] \cdot u_{1}^{4}=0$. Thus $\mathcal{N}(V)$ is a left $V$-subgroup of $V$ and, by anti-commutativity, a $V$-subgroup.

It has been shown that $\mathcal{E}(V)$ and $\mathcal{N}(V)$ are ideals of $V$ and $v^{4}=0$ for all $v$ in $\mathcal{N}(V)$. To prove $V=\mathcal{E}(V) \oplus \mathcal{N}(V)$ we note that $\mathcal{E}(V) \cap \mathcal{N}(V)=\{0\}$ and, by Proposition 5.1 (Proposition 4.1 also), a $w$ in $V$ is of the form $w^{4}+\left(w^{4}+w\right)$ where $w^{4}$ is in $\mathcal{E}(V)$. However, the map $\alpha$ taking $x$ in $V$ to $x^{4}$ is in $P_{0}(V)$ so that, by Theorem $5.4,\left(w^{4}+w\right) \alpha=\left(w^{4}+w\right)^{4}=w^{16}+w^{4}$ where, by Proposition 5.1 and 
Proposition 4.1, the last expression is 0 . Thus $w^{4}+w$ is in $\mathcal{N}(V)$ and the sum of the ideals $\mathcal{E}(V)$ and $\mathcal{N}(V)$ is $V$. Theorem 6.2 is proved.

If $V$ is a binary group and $U$ a $P_{0}(V)$ ring submodule of $V$, then Theorem 6.2 is applicable to the terminal ring $U$. A real enough question is whether $\mathcal{E}(U)$ and $\mathcal{N}(U)$ are ideals of $V$. It appears that for $\mathcal{E}(U)$ this may not be the case. For $\mathcal{N}(U)$ things are different.

THEOREM 6.3. If $V$ is a binary group and $U$ a $P_{0}(V) \operatorname{ring}$ submodule of $V$, then $\mathcal{N}(U)$ is an ideal of $V$.

Proof. Suppose $e$ is in $\mathcal{E}(U), u$ in $\mathcal{N}(U)$ and $\alpha$ in $P_{0}(V)$. Consider the map $\beta$ of $P_{0}(V)$ taking $v$ of $V$ to $(e v)(v \alpha)$. Since $U$ is a $P_{0}(V)$ ring submodule,

$$
(e(e+u))((e+u) \alpha)=(e+u) \beta=e \beta+u \beta=(e e)(e \alpha)+(e u)(u \alpha) .
$$

As $U$ is a $P_{0}(V)$ ring submodule and a non-associative ring the left hand side of (6.1) is $(e e+e u)(e \alpha+$ $u \alpha)$. Here, because $e e, e u, e \alpha$ and $u \alpha$ are all in the non-associative ring $U$, the left hand side of (6.1) is $(e e)(e \alpha)+(e e)(u \alpha)+(e u)(e \alpha)+(e u)(u \alpha)$. Thus, from $(6.1),(e e)(u \alpha)+(e u)(e \alpha)=0$ and since, $e u$ is in $\mathcal{E}(U) \cap \mathcal{N}(U)(=0)$ it follows that $e(u \alpha)=0$. It will be shown this is enough to prove $U$ is a $P_{0}(V)$-subgroup of $V$ so that, by Theorem 2.1, the theorem will be established.

Suppose there exists a $u_{1}$ in $U$ such that $u_{1} \gamma$ is not in $U$ for some $\gamma$ in $P_{0}(V)$. By Theorem 6.2, $u_{1} \gamma=u_{2}+u_{3}$, where $u_{2}(\neq 0)$ is in $\mathcal{E}(U)$ and $u_{3}$ in $\mathcal{N}(U)$. It follows readily that $u_{2}\left(u_{1} \gamma\right)=u_{2}$. But now with $e=u_{2}, u=u_{1}$ and $\alpha=\gamma$, we have a contradiction to what was proved above. It has been shown $U$ must be an ideal of $V$. Theorem 6.3 is proved.

Theorems 6.2 and 6.3 give considerable insight into $P_{0}(V)$ ring submodules of a binary group $V$. A lot more can be said when the ring submodule $U$ is minimal. The fact that elements of $P_{0}(V)$ distribute over $U$ can be used to show (not undertaken in this paper) either $U . U=\{0\}$ or $U$ is a simple Boolean ring.

\section{TERMINAL RINGS WITH NO ELEMENTS OF ORDER 2}

The definitions of solubility and nilpotency common to group settings have, after suitable generalization, been used more widely. In essence these concepts arise in the case of nearring groups (see [7]) and elsewhere. Indeed, $\Omega$-group solubility and nilpotency have gained importance (see for example [9] and [1]). Many questions can be asked. Certainly the $\Omega$-group solubility of $V$ implies its $P_{0}(V)$ solubility but finding conditions under which a converse holds is not easy. However, in spite of there being many elementary type $\Omega$-groups which are exceptions, reasonably general conditions supply us with a converse.

If $V$ is a terminal ring then, as observed in Theorem 6.2, $v^{4}=0$ for all $v$ in $\mathcal{N}(V)$. In view of this it seems natural to ask if there is a finite sequence $\{0\}=U_{0} \leq U_{1} \leq \cdots \leq U_{k}=\mathcal{N}(V)$ of ideals of $\mathcal{N}(V)$ (of $V$ ) such that $U_{i} . U_{i} \subseteq U_{i-1}$ for $i$ in $\{1, \ldots, k\}$ ? Questions of this sort have bearing on binary groups generally. Indeed such a sequence often allows us to obtain $\Omega$-group solubility (nilpotency) from the $P_{0}(V)$ solubility (nilpotency) of the binary group $V$. However, the question of when there is a series of this type is not always easily answered. In the case where $\mathcal{N}(V)$ has elements of order two it is not yet known if we can find such a series. On the other hand, when $V$ has no such element (here $V=\mathcal{N}(V)$ ) much can be said. In fact, in this situation, something much stronger than the existence of such a series can be supplied. The main step in proving the theorem involved is a lemma.

LEMMA 7.1. Suppose $V$ is a binary group and $U$ a $P_{0}(V)$ ring submodule of $V$ without elements of additive order two. If $a, b, c$ and $d$ are in $U$ and $\alpha$ and $\beta$ in $P_{0}(V)$, then $[(a b) \alpha][(c d) \beta]=0$ and $((((a b) \alpha) c) \beta) d=0$.

Proof. The map taking $v$ in $V$ to $[(v b) \alpha][(v d) \beta]$ is in $P_{0}(V)$ and, since $U$ is a $P_{0}(V)$ ring submodule of $V$, it follows that

$$
[((a+c) b) \alpha][((a+c) d) \beta]=[(a b) \alpha][(a d) \beta]+[(c b) \alpha][(c d) \beta] .
$$


Because $U$ is a $P_{0}(V)$ ring submodule and a non-associative ring the right hand side of this expression is

$$
[(a b) \alpha][(a d) \beta]+[(a b) \alpha][(c d) \beta]+[(c b) \alpha][(a d) \beta]+[(c b) \alpha][(c d) \beta],
$$

from which it follows that $[(a b) \alpha][(c d) \beta]=-[(c b) \alpha][(a d) \beta]$. In a similar fashion to that just employed (use the map taking $v$ in $V$ to $[(c v) \alpha][(a v) \beta]$ ) it can be shown that $[(c b) \alpha][(a d) \beta]=-[(c d) \alpha][(a b) \beta]$. Hence, $[(a b) \alpha][(c d) \beta]=[(c d) \alpha][(a b) \beta]$ which, on using the map taking $v$ in $V$ to $(v \alpha)(v \beta)$ supplies the fact that $[(a b) \alpha][(c d) \beta]=-[(a b) \alpha][(c d) \beta]$. The first statement of the lemma has been proved.

The second statement of the lemma is easily proved. In much the same way as was accomplished above (use the map taking $v$ in $V$ to $(v b) \alpha . v$ ) it can be shown that $0=[(a b) \alpha][(c d) \beta]=$ $-((((c d) \beta) b) \alpha) a$, so that with $c$ going to $a, d$ to $b, b$ to $c, a$ to $d, \beta$ to $\alpha$ and $\alpha$ to $\beta$, the desired conclusion is obtained. The lemma has been proved.

In the second paragraph of this section it was asked if a terminal ring had a certain series of ideals. It is now shown that when no elements of order two are involved something much stronger is possible.

THEOREM 7.2. If $V$ is a binary group and $U$ a $P_{0}(V)$ ring submodule of $V$ without elements of order two, then there exists a series $\{0\}=U_{0} \leq U_{1} \leq U_{2} \leq U_{3}=U$ of ideals of $V$ such that $U_{2} \cdot U_{2}=\{0\}$ and $\left(U . U_{i}\right) \cup\left(U_{i} . U\right) \subseteq U_{i-1}$ for $i=1,2$ and 3 .

Proof. For $v_{1}$ and $v_{2}$ in $U,\left(v_{1} v_{2}\right) P_{0}(V)$ is, by Theorem 2.1, an ideal of $V$ contained in $U$. Let $U_{2}$ be the $\operatorname{sum} \sum\left(v_{1} v_{2}\right) P_{0}(V)$ over all ideals of this kind. Certainly $U_{2}$ is contained in $U_{3}(=U)$. Now, for any such $v_{1}$ and $v_{2}$, it is the case that $\left(\left(v_{1} v_{2}\right) \alpha\right) v_{3}\left(v_{3}\right.$ in $U$ and $\alpha$ in $\left.P_{0}(V)\right)$ is in $U_{2}$. Thus, by Theorem 2.1, $\left(\left(\left(v_{1} v_{2}\right) \alpha\right) v_{3}\right) P_{0}(V)$ is an ideal of $V$ contained in $U_{2}$. Let $U_{1}$ be the sum $\sum\left(\left(\left(v_{1} v_{2}\right) \alpha\right) v_{3}\right) P_{0}(V)$ over all ideals of this kind (ie. with the $v_{i}, i=1,2,3$, taking values in $U$ and the $\alpha$ taking values in $P_{0}(V)$ ). Certainly $U_{1}$ is contained in $U_{2}$ but, as is now shown, $U_{1}$ and $U_{2}$ can be taken as those of the statement of Theorem 7.2 .

In proving this we first note that, from $U$ 's distributive laws, an element of $U_{2} \cdot U_{2}$, is a sum of elements of the form $\left(\left(a_{1} a_{2}\right) \alpha_{1}\right)\left(\left(a_{3} a_{4}\right) \alpha_{2}\right)$ where the $a_{i}$ are in $U$ and the $\alpha_{i}$ in $P_{0}(V)$. Since, by Lemma 7.1, such an element of $U$ is 0 , it follows that $U_{2} U_{2}=\{0\}$. Secondly, we note that, an element of $U U_{3}$ (of $U_{3} U$ ) is of the form $\left(b_{1} b_{2}\right) 1$ and is necessarily in $U_{2}$. Thirdly, we note that, from $U$ 's distributive laws, an element of $U_{2} U$ is a sum of elements of the form $\left(\left(\left(c_{1} c_{2}\right) \gamma\right) c_{3}\right) 1$ where the $c_{i}$ are in $U$ and the $\gamma$ in $P_{0}(V)$. Thus $U_{2} U$ is contained in $U_{1}$ and by anti-commutativity the same is true for $U U_{2}$. Finally, we note that, from $U$ 's distributive laws, an element of $U_{1} U$ is a sum of elements of the form $\left.\left(\left(\left(d_{1} d_{2}\right) \gamma_{1}\right) d_{3}\right) \gamma_{3}\right) d_{4}$ where the $d_{i}$ are in $U$ and the $\gamma_{i}$ in $P_{0}(V)$. By Lemma 7.1, such an element is 0 . Thus $U_{1} U$ has to be in $\{0\}$ and by anti-commutativity the same is true for $U U_{1}$. Theorem 7.2 is proved.

Theorem 7.2 tells us that the terminal ring $U$ (see Theorem 5.3) is rather restricted. In the next section it will be seen that finite $U$ are often even more basic (see Theorem 8.3). A definition along these lines needs to be made. A basic terminal ring $V$ is taken as one with an ideal $W$ such that $V V \subseteq W$ and $(W V) \cup(V W)=\{0\}$. A characterization of anti-commutative rings, which are basic terminal rings and without elements of order two, follows. This result will be used in sections eleven, twelve and thirteen.

THEOREM 7.3. If $V$ is an anti-commutative ring without elements of order two, then the following are equivalent:

(i) $V$ is associative,

(ii) $(a b) c=0$ for all $a, b$ and $c$ in $V$,

(iii) $V$ is an associative terminal ring and

(iv) $V$ is a basic terminal ring.

Proof. Proving the equivalence of (ii) to (iv) and showing (ii) implies (i) are rather elementary matters. Their proofs are omitted. It will be shown that (i) implies (ii). Anti-commutativity, the right 
distributive law and associativity imply $(v b) v=-(b v) v=-b(v v)=0$ for all $v$ in $V$. Thus,

$$
((a+c) b)(a+c)=0=(a b) a+(a b) c+(c b) a+(c b) c=(a b) c+(c b) a
$$

so that $(a b) c=-(c b) a=(b c) a=-a(b c)$. Because $(a b) c=a(b c)$, it can be concluded that $(a b) c=0$. Theorem 7.3 is proved.

A feature of a basic $P_{0}(V)$ ring submodule $W$ of a binary group $V$ is that an ideal $U$ of $W$, having $W W \subseteq U$ and $U W=\{0\}$, can in fact be taken as an ideal of $V$.

PROPOSITION 7.4. If $W$ is a basic $P_{0}(V)$ ring submodule of the binary group $V$, then there exists an ideal $U(\leq W)$ of $V$ such that $W W \subseteq U$ and $U W=\{0\}$.

Proof. As in the proof of Theorem 7.2, we see that the sum $\sum\left(w_{1} w_{2}\right) P_{0}(V)$ over all $w_{i}, i=1,2$, in $W$ is an ideal of $V$ contained in $W$. We take this to be $U$. Clearly, $W W \subseteq U$. With $\alpha$ in $P_{0}(V)$, the map taking $v$ in $V$ to $(v \alpha) v$ is in $P_{0}(V)$. Because $W$ is a $P_{0}(V)$ ring submodule of $V$, this element of $P_{0}(V)$ supplies the fact that $\left(u_{1} u_{2}\right) \alpha u_{3}+\left(u_{3} \alpha\right)\left(u_{1} u_{2}\right)=0\left(u_{i}, i=1,2,3\right.$, in $\left.W\right)$. As $\left(u_{3} \alpha\right)\left(u_{1} u_{2}\right)=-\left(u_{1} u_{2}\right)\left(u_{3} \alpha\right)=0$, it follows that $\left(u_{1} u_{2}\right) \alpha u_{3}=0$. The distributive laws imply $U W=\{0\}$. Proposition 7.4 is proved.

In the next section it will be seen that many finite terminal rings are basic.

\section{CERTAIN FINITE TERMINAL RINGS CANNOT OCCUR}

Finite basic terminal rings of odd order are in reality rather rudimentary associative rings (see Theorem 7.3). However, if Theorem 7.2 is taken into consideration, it seems natural to ask if there are any finite terminal rings of odd order which are not basic? The present section does not answer this. Instead results are supplied that tend to justify the need to look into this. What is proved here (Corollary 8.4) is that finite terminal rings of order $p_{1}^{n_{1}} p_{2}^{n_{2}} \ldots p_{k}^{n_{k}}$ (the $p_{i}$ distinct odd primes and the $n_{i}$ integers $\geq 1$ and $\leq 6$ ) are in fact basic. In section ten it will be shown there is in fact a non-basic terminal ring of order $p^{7}$ ( $p$ an odd prime). Things start with a rather elementary proposition. Its corollary will help with the proof of the theorem that follows.

PROPOSITION 8.1. Suppose $V$ is a binary group and $U$ a $P_{0}(V)$ ring submodule of $V$ without elements of order two. If $u$ is in $U$, then the ideal $I d(u)$ of $V$ generated by $u$ is such that $\operatorname{Id}(u) \cdot \operatorname{Id}(u)=\{0\}$.

Proof. It readily follows from Theorem 2.1 that $u P_{0}(V)=I d(u)$. Thus an element of $\operatorname{Id}(u) \cdot \operatorname{Id}(u)$ is of the form $(u \alpha)(u \beta)$ ( $\alpha$ and $\beta$ in $P_{0}(V)$ ). Consider the element of $P_{0}(V)$ taking $v$ in $V$ to $(v \alpha)(v \beta)$. The ring module nature of $U$ supplies the fact that

$$
((u+u) \alpha)((u+u) \beta)=(u \alpha)(u \beta)+(u \alpha)(u \beta)=(u \alpha+u \alpha)(u \beta+u \beta),
$$

where the right hand side is $[(u \alpha)(u \beta)] 4$. Thus $[(u \alpha)(u \beta)] 4=[(u \alpha)(u \beta)] 2$ and $(u \alpha)(u \beta)=\{0\}$ so that $\operatorname{Id}(u) \cdot \operatorname{Id}(u)=\{0\}$. Proposition 8.1 is proved.

From Theorem 5.4 and Proposition 8.1 we have:

COROLLARY 8.2. Suppose $V$ is a terminal ring without elements of order two. If $u$ is in $V$, then $\operatorname{Id}(u) \cdot \operatorname{Id}(u)=\{0\}$.

A real enough impression conveyed by the theorem that follows is that finite non-basic terminal rings of odd order must be rare.

THEOREM 8.3. A terminal ring $V$ having order $p^{n}$ ( $p$ an odd prime) is basic whenever the integer $n$ $(\geq 1)$ is $\leq 6$.

Proof. Suppose $V$ of the type indicated is not basic. Elements $u, v$ and $w$ of $V$ can be found such that $(u v) w \neq 0$ (see Theorem 7.3). As $V$ is anti-commutative, anti-associative and such that $b b=0$ for all $b$ in $V$, there is no real difficulty in showing the subgroup $\langle u, v, w, u v, u w, v w,(u v) w\rangle$ (=W say) of $V$ is a terminal subring. Since $(u v) w \neq 0$ this terminal subring is non-basic. Also anti-commutativity, anti-associativity and the fact that $b b=0$ for all $b$ in $W$, imply $\langle u v, u w, v w,(u v) w\rangle(=A$ say) is an ideal of $W$. 
It is now shown that $W / A$ cannot be generated by a subset of $\{u+A, v+A, w+A\}$ of less than three elements. From the symmetry of the situation proving $\langle u+A, v+A\rangle$ is $\neq W / A$ is sufficient for this. If $\langle u+A, v+A\rangle$ were to be $=W / A$ then, as the ideals $I d(u)$ and $I d(v)$ of $W$ generated by $u$ and $v$ are such that $A \leq I d(u)+I d(v)$, we would have $I d(u)+I d(v)=W$. Here, with $I d(u) \cap I d(v)=B$, $W / B$ is a direct sum $(I d(u) / B) \oplus(I d(v) / B)$ of two null groups (see Corollary 8.2) so that $W W \subseteq B$. However, by Corollary 8.2 , the fact that $B W \subseteq B \cdot I d(u)+B \cdot I d(v)=\{0\}$ yields the contradiction, $W$ must be basic. Thus $W / A$ is generated by $u+A, v+A$ and $w+A$ and not by a two or one element subset. This means $|W / A| \geq p^{3}$ and, as $|W| \leq p^{6}$, it therefore follows that $|A| \leq p^{3}$.

The fact that $|A| \leq p^{3}$ means a subset that additively generates $A$ contains a subset $S$ with $|S| \leq 3$ and $\langle S\rangle=(A,+)$. Thus, even if some of the elements of $\{u v, u w, v w,(u v) w\}$ coincide, there is a subset $S_{1}$ of this set with $\left|S_{1}\right| \leq 3$ and $\left\langle S_{1}\right\rangle=(A,+)$. A consequence of this is that one of the subsets

$$
\{u v, u w,(u v) w\},\{u v, v w,(u v) w\},\{u w, v w,(u v) w\}
$$

or $\{u v, u w, v w\}$ additively generate $A$. Suppose $\{u v, u w, v w\}$ is such a subset. Here, either two elements of it additively generate $A$ or, because $A$ has order $p^{3},(A,+)$ is of the form $C_{p} \oplus C_{p} \oplus C_{p}$ (here $C_{k}$ with $k \geq 1$ an integer is the cyclic group of order $k$ ). In the $C_{p} \oplus C_{p} \oplus C_{p}$ case $(u v) w$ is a non-zero element of $A$ and, from this it is easy enough to see, that one of the subsets of (8.1) generate $(A,+)$. It is now shown how this leads to a contradiction. As is not hard to see, the symmetry of the situation allows us to assume $\{u v, u w,(u v) w\}$ additively generates $A$. Thus $v w$ is in $\langle u v, u w,(u v) w\rangle$ and $u\langle u v, u w,(u v) w\rangle \neq\{0\}$. This is contrary to the fact that $u(u v)=u(u w)=u((u v) w)=0$ (use the terminal nature of $V)$. A contradiction has been arrived at and Theorem 8.3 is proved.

In a finite non-associative ring $V$, the distributive laws imply the Sylow subgroups of $(V,+)$ are ideals. As a consequence $V$ is a direct sum of these ideals. By Theorem 8.3, a proof of the corollary announced at the outset of this section has been achieved. We have:

COROLLARY 8.4. A terminal ring $V$ having order $p_{1}^{n_{1}} p_{2}^{n_{2}} \ldots p_{k}^{n_{k}}\left(k \geq 1\right.$ an integer and the $p_{i}$ distinct odd primes with $n_{i}$ integers $\geq 1$ and $\leq 6$ ) is basic.

\section{SAC-DECOMPOSITION OF ANTI-COMMUTATIVE RINGS}

In order to proceed further in the study of terminal rings some general theory is required. That is what this section supplies. The last section raised the question of whether there are finite non-basic terminal rings of odd order. Theory obtained here will allow us to answer this. It will also be of considerable help in classifying terminal rings of order $p^{n}$ ( $p$ an odd prime and $n \geq 1$ an integer $\leq 4$ ). What is now undertaken is supplying a way in which an anti-commutative ring $V$ can be specified. For this we need the subgroup $\operatorname{ker}_{2}(V)$ of all elements of $V$ of order two (kernel of the map taking $v$ in $V$ to $v 2$ ).

We come now to defining what might be called a sub-anti-commutative decomposition of a finite abelian group $V$. Such a decomposition will simply be called an sac-decomposition of $V$. Here if $n \geq 1$ is an integer, then a sac-decomposition of $V$ (more precisely a (sac) $)_{n}$-decomposition of $V$ ) is a pair $(\Lambda, \mu)$ where $\Lambda$ is a certain type of subset of $V^{*}$ and $\mu$ a certain type of function of $\Lambda \times \Lambda$ into $V . \Lambda$ is required to be $n$ distinct elements $a_{1}, \ldots, a_{n}$ of $V^{*}$ and $V=\left\langle a_{1}\right\rangle \oplus\left\langle a_{2}\right\rangle \oplus \cdots \oplus\left\langle a_{n}\right\rangle\left\langle\left\langle a_{i}\right\rangle\right.$ is the cyclic subgroup of $V$ generated by $\left.a_{i}\right) . \mu$ is required to be a function of $\Lambda \times \Lambda$ into $V$ with the following properties:

(i) $\left(a_{i}, a_{j}\right)(i>j$ in $\{1, \ldots, n\})$ is mapped by $\mu$ to $\left(a_{i}, a_{j}\right) \mu$ where the only requirement is that the order $\left|\left(a_{i}, a_{j}\right) \mu\right|$ of $\left(a_{i}, a_{j}\right) \mu$ divides $\left|a_{i}\right|$ and divides $\left|a_{j}\right|$,

(ii) $\left(a_{i}, a_{i}\right) \mu$ is in $\operatorname{ker}_{2}(V)$ for $i=1, \ldots, n$ and

(iii) $\left(a_{i}, a_{j}\right) \mu$ is taken as $-\left(a_{j}, a_{i}\right) \mu$ for $i<j$ in $\{1, \ldots, n\}$.

sac-decompositions of a finite abelian group $V$ have been defined and with $(\Lambda, \mu)$ such a decomposition our intension now becomes to show that the function $\mu$ of $\Lambda \times \Lambda$ into $V$ can be extended in a unique way to one $e(\mu)$ of $V \times V$ into $V$ in such a way that, under $e(\mu), V$ is an anti-commutative ring. 
Suppose $V$ is a finite abelian group, $(\Lambda, \mu)$ a (sac) $)_{n}$-decomposition of $V, \Lambda=\left\{a_{1}, \ldots, a_{n}\right\}$ and $b$ and $c$ elements of $V$. If $b$ (if $c$ ) can be written as $a m_{1}+\cdots+a_{n} m_{n}\left(\right.$ as $\left.a_{1} k_{1}+\cdots+a_{n} k_{n}\right)$ where $m_{i}$ and $k_{i}$, $i=1, \ldots, n$, are integers, then the product $(b, c) e(\mu)$ of $b$ and $c$ is defined to be $\sum_{i=1}^{n} \sum_{j=1}^{n}\left(a_{i}, a_{j}\right) \mu m_{i} k_{j}$. There is no ambiguity in this definition since if $b=a_{1} m_{1}^{\prime}+\cdots+a_{n} m_{n}^{\prime}$ and $c=a_{1} k_{1}^{\prime}+\cdots+a_{n} k_{n}^{\prime}$ where the $m_{i}^{\prime}$ and $k_{j}^{\prime}$ are integers, then $m_{i} \equiv m_{i}^{\prime} \bmod \left|a_{i}\right|$ and $k_{j} \equiv k_{j}^{\prime} \bmod \left|a_{j}\right|$ so that $m_{i} k_{j} \equiv m_{i}^{\prime} k_{j}^{\prime}$ $\bmod$ g.c.d $\left(\left|a_{i}\right|,\left|a_{j}\right|\right)$ and $m_{i} k_{j} \equiv m_{i}^{\prime} k_{j}^{\prime} \bmod \left|\left(a_{i}, a_{j}\right) \mu\right|$.

PROPOSITION 9.1. If $V$ is a finite abelian group and $(\Lambda, \mu)$ a (sac) $)_{n}$-decomposition of $V$, then $e(\mu)$ is the unique extension of $\mu$ from $\Lambda \times \Lambda$ to $V \times V$ making $V$ into an anti-commutative ring.

Proof. Suppose $\Lambda=\left\{a_{1}, \ldots, a_{n}\right\}$ and $b=a_{1} m_{1}+\cdots+a_{m} m_{n}$ and $c=a_{1} k_{1}+\cdots+a_{n} k_{n}$ are in $V$ with the $m_{i}$ and $k_{i}$ being integers. If $\mu^{\prime}$ is an extension of $\mu$ to $V \times V$ making $V$ into an anti-commutative ring, then the distributive laws imply that $(b, c) \mu^{\prime}=\sum_{i=1}^{n} \sum_{j=1}^{n}\left(a_{i}, a_{j}\right) \mu m_{i} k_{j}$. Thus $\mu^{\prime}=e(\mu)$. It only remains to show that, under $e(\mu), V$ is an anti-commutative ring. If $d=a_{1} l_{1}+\cdots+a_{n} l_{n}$ ( $l_{1}$ to $l_{n}$ integers), then $(d, b+c) e(\mu)=\sum_{i=1}^{n} \sum_{j=1}^{n}\left(a_{i}, a_{j}\right) \mu l_{i}\left(m_{j}+k_{j}\right)$ where the right hand side is

$$
\sum_{i=1}^{n} \sum_{j=1}^{n}\left(a_{i}, a_{j}\right) \mu l_{i} m_{j}+\sum_{i=1}^{n} \sum_{j=1}^{n}\left(a_{i}, a_{j}\right) \mu l_{i} k_{j}=(d, b) e(\mu)+(d, c) e(\mu) .
$$

As a similar argument shows that $(b+c, d) e(\mu)=(b, d) e(\mu)+(c, d) e(\mu)$, it only remains to show $e(\mu)$ satisfies the anti-commutative law. To see this we note the definition of $\mu$ supplies the fact that

$$
(b, c) e(\mu)=\sum_{i=1}^{n} \sum_{j=1}^{n}\left(a_{i}, a_{j}\right) \mu m_{i} k_{j}=\sum_{j=1}^{n} \sum_{i=1}^{n}\left[-\left(a_{j}, a_{i}\right) \mu\right] k_{j} m_{i},
$$

where the right hand side is $-[(c, b) e(m u)]$. Proposition 9.1 is proved.

The proof of Proposition 9.1 was very straightforward. Two further results of an elementary nature are now given. The first is a proposition showing that for finite non-zero anti-commutative rings there are in general many ways in which the binary operation is an $e(\mu)$ ( $\mu$ as in Proposition 9.1). The second is also a proposition. It supplies information as to when a group isomorphism between finite abelian groups is in fact an isomorphism of anti-commutative rings.

PROPOSITION 9.2. Suppose $V$ is a non-zero finite anti-commutative ring with additive group a direct sum $\left\langle a_{1}\right\rangle \oplus \cdots \oplus\left\langle a_{n}\right\rangle$ of the non-zero cyclic subgroups $\left\langle a_{i}\right\rangle\left(a_{i} \in V^{*}\right)$. If $\Lambda=\left\{a_{1}, \ldots, a_{n}\right\}$ and $\mu$ the function of $\Lambda \times \Lambda$ into $V$ defined by $\left(a_{i}, a_{j}\right) \mu=a_{i} a_{j}((i, j) \in\{1, \ldots, n\} \times\{1, \ldots, n\})$, then $(\Lambda, \mu)$ is a (sac) ${ }_{n}$-decomposition of $V$ and $e(\mu)$ is the binary operation of $V$.

Proof. It is not difficult to see $\mu$ satisfies (i), (ii) and (iii) of the definition of (sac) ${ }_{n}$-decomposition. It is now clear from Proposition 9.1 that $e(\mu)$ is the binary operation of $V$. Proposition 9.2 holds.

PROPOSITION 9.3. Suppose $V_{i}, i=1,2$, are two finite abelian groups, $V_{1}=\left\langle a_{1}\right\rangle \oplus \cdots \oplus\left\langle a_{n}\right\rangle$ ( $n \geq 1$ and $a_{i}$, $i=1, \ldots, n$, elements of $\left.V_{1}^{*}\right), V_{2}=\left\langle b_{1}\right\rangle \oplus \cdots \oplus\left\langle b_{n}\right\rangle\left(b_{1}, \ldots, b_{n}\right.$, elements of $\left.V_{2}^{*}\right)$ and $\tau$ is an isomorphism of $V_{1}$ onto $V_{2}$ with the property that $a_{i} \tau=b_{i}$ for $i=1, \ldots, n$. If $\left(\Lambda_{i}, \mu_{i}\right)$ are $(\mathrm{sac})_{n}$-decompositions of $V_{i}, i=1,2$, with $\Lambda_{1}=\left\{a_{1}, \ldots, a_{n}\right\}, \Lambda_{2}=\left\{b_{1}, \ldots, b_{n}\right\}$ and $\left(a_{j}, a_{k}\right) \mu_{1} \tau=\left(b_{j}, b_{k}\right) \mu_{2}$ for all $j$ and $k$ in $\{1, \ldots, n\}$, then $\tau$ is an anti-commutative ring isomorphism of $V_{1}$ (multiplication given by $e\left(\mu_{1}\right)$ ) onto $V_{2}$ (multiplication given by $e\left(\mu_{2}\right)$ ).

Proof. Proving $\tau$ is as indicated amounts to showing $(c, d) e\left(\mu_{1}\right) \tau\left(c\right.$ and $d$ in $\left.V_{1}\right)$ is $(c \tau, d \tau) e\left(\mu_{2}\right)$. Without going into detail it can be seen that, with $c=a_{1} m_{1}+\cdots+a_{n} m_{n}$ and $d=a_{1} l_{1}+\cdots+a_{n} l_{n}$ (the $m_{i}$ and $l_{j}$ integers), we have

$$
(c, d) e\left(\mu_{1}\right) \tau=\left(\sum_{i=1}^{n} \sum_{j=1}^{n}\left(a_{i}, a_{j}\right) \mu_{1} m_{i} l_{j}\right) \tau=\sum_{i=1}^{n} \sum_{j=1}^{n}\left(b_{i}, b_{j}\right) \mu_{2} m_{i} l_{j}=(c \tau, d \tau) e\left(\mu_{2}\right)
$$

and Proposition 9.3 holds.

Results of this section are now made use of to supply a rather noteworthy terminal ring. 


\section{A NON-BASIC TERMINAL RING OF ORDER $P^{7}$ (P ODD)}

Theorem 8.3 raises a question. Does there exist a finite non-basic terminal ring of order $p^{n}$ ( $p$ an odd prime and $n \geq 1$ an integer)? The answer to this question is that such a terminal ring $V$ can be found. We now move into providing results affirming this. The material supplied is more illuminating than just the proof that $V$ exists. This is because of Theorem 8.3. What is supplied is a $V$ of order $p^{7}$.

Things start by specifying the $(V,+)$ we will be considering. It is determined by being able to take within it seven elements $u_{i}, i=1, \ldots, 7$, of additive order $p$ with $(V,+)=\oplus_{i=1}^{7}\left\langle u_{i}\right\rangle$. Such a $(V,+)$ can be considered as the additive group of the seven dimensional vector space over the field $Z_{p}$ of $p$ elements. It will make sense to denote it by $\mathcal{V}_{p}(7)$. The idea now becomes to define a (sac) $)_{7}$-decomposition $\left(\left\{u_{1}, \ldots, u_{7}\right\}, \mu\right)$ on $V$ having the property that $V$ is, with respect to $e(\mu)$, a non-basic terminal ring. Thus, in accordance with the fact that $\left(u_{i}, u_{i}\right) \mu$ must be 0 for $i=1, \ldots, 7$ and the fact that $\left(u_{j}, u_{i}\right) \mu$ must be $-\left(u_{i}, u_{j}\right) \mu$ for $i<j(\{i, j\} \subseteq\{1, \ldots 7\})$ we need only indicate the value of $\left(u_{i}, u_{j}\right) \mu$ for $i<j$. There are twenty one different $\{i, j\}$ needing consideration. In listing these, five categories that respectively deal with $i=1,2,3,4$ and $i$ in $\{5,6\}$, are provided. The first three categories will in turn introduce integers $k_{1}, k_{2}$ and $k_{3}$ (each $\geq 1$ and coprime to $p$ ). Values for these integers will soon be given that make our anti-commutative ring $V$ (multiplication $e(\mu)$ ) non-basic and terminal. A (sac) $)_{7}$-decomposition $\left(\left\{u_{1}, \ldots, u_{7}\right\}, \mu\right)$ of $\mathcal{V}_{p}(7)$ of the type now supplied will be called a $\operatorname{sac}\left(k_{1}, k_{2}, k_{3}\right)$-decomposition of $(V,+)$ (ie. of $\left.\mathcal{V}_{p}(7)\right)$. The listing of the $\left(u_{i}, u_{j}\right) \mu$ is as follows:

The six $\left(u_{i}, u_{j}\right) \mu$ with $i=1$ are given by,

$$
\left(u_{1}, u_{j}\right) \mu=0 \quad \text { for } j=2, \ldots, 6 \quad \text { and } \quad\left(u_{1}, u_{7}\right) \mu=u_{4} k_{1} .
$$

The five $\left(u_{i}, u_{j}\right) \mu$ with $i=2$ are given by,

$$
\left(u_{2}, u_{3}\right) \mu=\left(u_{2}, u_{4}\right) \mu=\left(u_{2}, u_{5}\right) \mu=\left(u_{2}, u_{7}\right) \mu=0 \quad \text { and } \quad\left(u_{2}, u_{6}\right) \mu=u_{4} k_{2} .
$$

The four $\left(u_{i}, u_{j}\right) \mu$ with $i=3$ are given by

$$
\left(u_{3}, u_{4}\right) \mu=\left(u_{3}, u_{6}\right) \mu=\left(u_{3}, u_{7}\right) \mu=0 \quad \text { and } \quad\left(u_{3}, u_{5}\right) \mu=u_{4} k_{3} .
$$

The three $\left(u_{i}, u_{j}\right) \mu$ with $i=4$ are given by

$$
\left(u_{4}, u_{5}\right) \mu=\left(u_{4}, u_{6}\right) \mu=\left(u_{4}, u_{7}\right) \mu=0 .
$$

The two (one) $\left(u_{i}, u_{j}\right) \mu$ where $i=5$ (where $i=6$ ) are given by

$$
\left(u_{5}, u_{6}\right) \mu=u_{1},\left(u_{5}, u_{7}\right) \mu=u_{2} \quad \text { and } \quad\left(u_{6}, u_{7}\right) \mu=u_{3} .
$$

With this definition of $\operatorname{sac}\left(k_{1}, k_{2}, k_{3}\right)$-decomposition behind us, something seeming rather meaningful can be supplied.

THEOREM 10.1. If $V$ is an anti-commutative ring with additive group $\mathcal{V}_{p}(7)$, then $V$ is a non-basic terminal ring if, and only if, $(V,+)$ has a $\operatorname{sac}(1, p-1,1)$-decomposition $\left(\left\{u_{1}, \ldots, u_{7}\right\}, \mu\right)$ with $e(\mu)$ the multiplication in $V$.

Proof. It is first shown that if $V$ is a non-basic terminal ring, then the sac $(1, p-1,1)$-decomposition $\left(\left\{u_{1}, \ldots, u_{7}\right\}, \mu\right)$ of $(V,+)$ exists.

According to Theorem 7.3, there must exist $u_{5}, u_{6}$, and $u_{7}$ in $V$ such that $\left(u_{5} u_{6}\right) u_{7} \neq 0$. It will be shown that with $u_{1}=u_{5} u_{6}, u_{2}=u_{5} u_{7}, u_{3}=u_{6} u_{7}, u_{4}=\left(u_{5} u_{6}\right) u_{7}$ and $\mu$ the function of $\left\{u_{1}, \ldots, u_{7}\right\} \times\left\{u_{1}, \ldots, u_{7}\right\}$ into $V$ given by $\left(u_{i}, u_{j}\right) \mu=u_{i} u_{j}(\{i, j\} \subseteq\{1, \ldots, 7\}),\left(\left\{u_{1}, \ldots, u_{7}\right\}, \mu\right)$ is a $\operatorname{sac}(1, p-1,1)$-decomposition of $(V,+)$ where multiplication in $V$ is $e(\mu)$. In this direction things start by showing $\left|u_{i}\right|=p, i=1, \ldots, 7$, and $(V,+)=\oplus_{i=1}^{7}\left\langle u_{i}\right\rangle$. An aspect of this (details omitted) is to note that the definition of the $u_{i}, i=1, \ldots, 7$, and the terminal nature of $V$ imply the subgroup $\left\langle u_{1}, \ldots, u_{7}\right\rangle$ of $(V,+)$ is a terminal subring of $V$. It cannot be basic as $\left(u_{5} u_{6}\right) u_{7} \neq 0$. A consequence of Theorem 8.3 and the fact that $|(V,+)|=p^{7}$ is that $\left\langle u_{1}, \ldots, u_{7}\right\rangle=(V,+)$. As $(V,+)=\mathcal{V}_{p}(7)$ and $(V,+)$ is generated by the seven elements $u_{i}, i=1, \ldots, 7$, we see $\left|u_{i}\right|=p$ and $(V,+)=\oplus_{i=1}^{7}\left\langle u_{i}\right\rangle$. Things now move into showing that if in (10.1) to (10.5), $k_{1}=1, k_{2}=p-1$ and $k_{3}=1$, then these equations are satisfied. 
The manner in which $u_{1}, u_{2}$ and $u_{3}$ are defined ensure (10.5) holds. The terminal nature of $V$ along with the definition of $u_{2}, u_{3}$ and $u_{4}$ imply $\left(u_{1}, u_{j}\right) \mu=0$ for $j$ in $\{2,3,4\}$. Also the definition of $u_{1}$ and the terminal nature of $V$ implies $\left(u_{1}, u_{5}\right) \mu=\left(u_{1}, u_{6}\right) \mu=0$. On also taking into consideration the definition of $u_{4}$ we see that (10.1) holds with $k_{1}=1$. Without going into unnecessary detail, definitions of the $u_{i}, i=1, \ldots, 7$, are seen to yield the fact that $\left(u_{2}, u_{j}\right) \mu=0$ for $j \in\{3,4,5,7\}$. Also $u_{2} u_{6}=\left(u_{5} u_{7}\right) u_{6}=-\left(u_{5} u_{6}\right) u_{7}\left(V\right.$ is terminal) so that (10.2) holds with $k_{2}=p-1$. Again the nature of the $u_{i}$ and $V$ implies $\left(u_{3}, u_{j}\right) \mu=0$ for $j \in\{4,6,7\}$ while $u_{3} u_{5}=\left(u_{6} u_{7}\right) u_{5}=\left(u_{5} u_{6}\right) u_{7}$. Thus (10.3) holds when $k_{3}$ is taken as 1 . Furthermore $\left(u_{4}, u_{j}\right) \mu=0$ for $j$ in $\{5,6,7\}$. It has been shown that with $k_{1}=1$, $k_{2}=p-1$ and $k_{3}=1,(10.1)$ to $(10.5)$ hold. Thus $\left(\left\{u_{1}, \ldots, u_{7}\right\}, \mu\right)$ is a $\operatorname{sac}(1, p-1,1)$-decomposition of $(V,+)$. The multiplication in $V$ is $e(\mu)$ (see Proposition 9.2). It remains to show that when such a decomposition exists, the $V$ (multiplication $e(\mu))$ is a non-basic terminal ring.

Suppose the $\operatorname{sac}(1, p-1,1)$ decomposition $\left(\left\{u_{1}, \ldots, u_{7}\right\}, \mu\right)$ of $(V,+)$ exists and is such that the product $w_{1} w_{2}$ of $w_{i}, i=1,2$, of $V$ is $\left(w_{1}, w_{2}\right) e(\mu)$. From Proposition 9.1, it follows that $V$ has become an anti-commutative ring. We now set about showing it is anti-associative. A substantial part of this will be to show that $\left(u_{i} u_{j}\right) u_{k}=-u_{i}\left(u_{j} u_{k}\right)$ for all $i, j$ and $k$ in $\{1, \ldots, 7\}$. Three cases occur.

CASE 1. With $S=\left\{u_{1}, u_{2}, u_{3}, u_{4}\right\}$ it is shown the assumption that $\left\{u_{i}, u_{j}, u_{k}\right\} \cap S \neq \varnothing \operatorname{implies}\left(u_{i} u_{j}\right) u_{k}=$ $0=u_{i}\left(u_{j} u_{k}\right)$.

Here reasonably careful examination of (10.1) to (10.5) along with the anti-commutative ring nature of $V$ implies $V . V \subseteq\langle S\rangle,\langle S\rangle .\langle S\rangle=\{0\},(\langle S\rangle . V) \cup(V .\langle S\rangle) \subseteq\left\langle u_{4}\right\rangle$ and $\left(\left\langle u_{4}\right\rangle . V\right) \cup\left(V .\left\langle u_{4}\right\rangle\right)=\{0\}$. This means that if an element of $\left\{u_{i}, u_{j}\right\}$ were to be in $S$, then $u_{i} u_{j}$ would be in $\left\langle u_{4}\right\rangle$ and $\left(u_{i} u_{j}\right) u_{k}$ would be 0 . However, if $u_{k}$ were to be in $S$, $\left(u_{i} u_{j}\right) u_{k}$ would be in $S . S$ and again be 0 . Thus, if an element of $\left\{u_{i}, u_{j}, u_{k}\right\}$ is in $S$, then $\left(u_{i} u_{j}\right) u_{k}=0$. From this and the fact that $u_{i}\left(u_{j} u_{k}\right)=-\left(u_{j} u_{k}\right) u_{i}$ it follows that, if an element of $\left\{u_{i}, u_{j}, u_{k}\right\}$ where to be in $S$, then $u_{i}\left(u_{j} u_{k}\right)=0$. Thus, if an element of $\left\{u_{i}, u_{j}, u_{k}\right\}$ is in $S$, then $\left(u_{i} u_{j}\right) u_{k}=0=u_{i}\left(u_{j} u_{k}\right)$.

CASE 2. Here we prove that if $\left\{u_{i}, u_{j}, u_{k}\right\}$ (assumed to be $\subseteq\left\{u_{5}, u_{6}, u_{7}\right\}$ ) is a two or one element subset of $V$, then $\left(u_{i} u_{j}\right) u_{k}=0=u_{i}\left(u_{j} u_{k}\right)$.

Since $u_{i}\left(u_{j} u_{k}\right)=-\left(u_{j} u_{k}\right) u_{i}$, this will follow if it is shown that for $\left|\left\{u_{i}, u_{j}, u_{k}\right\}\right| \leq 2$ we have $\left(u_{i} u_{j}\right) u_{k}=0$. If $u_{i}=u_{j}$, then $\left(u_{i} u_{j}\right) u_{k}=0$. Thus, it can be assumed either $u_{i}=u_{k}$ or $u_{j}=u_{k}$ and because $\left(u_{i} u_{j}\right) u_{k}=-\left(u_{j} u_{i}\right) u_{k}$ it is only required it be shown that $\left(u_{i} u_{j}\right) u_{i}=0$. For $i=5$ and $j$ taken respectively as 5,6 and $7, u_{i} u_{j}$ becomes $0, u_{1}$ and $u_{2}$ (see (10.5)). Because $\left\{u_{1}, u_{2}\right\} u_{5}=\{0\}$ (see (10.1) and (10.2)), $\left(u_{i} u_{j}\right) u_{i}=0$ when $i=5$. For $i=6$ and $j$ taken respectively as 5,6 and $7, u_{i} u_{j}$ becomes $-u_{1}, 0$ and $u_{3}$ (see (10.5)). Because $\left\{u_{1}, u_{3}\right\} u_{6}=\{0\}$ (see (10.1) and (10.3)), $\left(u_{i} u_{j}\right) u_{i}=0$ when $i=6$. For $i=7$ and $j$ taken respectively as 5,6 and $7 u_{i} u_{j}$ becomes $-u_{2},-u_{3}$ and 0 (see (10.5)). Because $\left\{u_{2}, u_{3}\right\} u_{7}=\{0\}$ (see (10.2) and (10.3)), $\left(u_{i} u_{j}\right) u_{i}=0$ when $i=7$. Thus, if $\{i, j, k\}$ has order $\leq 2$ and is contained in $\{5,6,7\}$, then it must follow that $\left(u_{i} u_{j}\right) u_{k}=0=u_{i}\left(u_{j} u_{k}\right)$.

CASE 3. This is the final step in proving $\left(u_{i} u_{j}\right) u_{k}=-u_{i}\left(u_{j} u_{k}\right)(\{i, j, k\} \subseteq\{1, \ldots, 7\})$.

It will deal with $\{i, j, k\}$ coinciding with $\{5,6,7\}$. More work goes into this case than that of 1 or 2. There are six possibilities that must be checked out. These are $\left(u_{5} u_{6}\right) u_{7}$ ((a) say), $\left(u_{5} u_{7}\right) u_{6}((\mathrm{~b})$ say), $\left(u_{6} u_{5}\right) u_{7}$ ((c) say), $\left(u_{7} u_{6}\right) u_{5}$ (d), $\left(u_{6} u_{7}\right) u_{5}$ (e) and $\left(u_{7} u_{5}\right) u_{6}$ (f). For (a)

$$
\left(u_{5} u_{6}\right) u_{7}=u_{1} u_{7}=u_{4} \quad \text { while } \quad u_{5}\left(u_{6} u_{7}\right)=u_{5} u_{3}=-u_{4} \text {. }
$$

For (b)

$$
\left(u_{5} u_{7}\right) u_{6}=u_{2} u_{6}=-u_{4} \quad \text { while } \quad u_{5}\left(u_{7} u_{6}\right)=u_{5}\left(-u_{3}\right)=u_{4}
$$

For $(\mathrm{c})$

For $(\mathrm{d})$

$$
\left(u_{6} u_{5}\right) u_{7}=\left(-u_{1}\right) u_{7}=-u_{4} \quad \text { while } \quad u_{6}\left(u_{5} u_{7}\right)=u_{6} u_{2}=u_{4}
$$

For (e)

$$
\left(u_{7} u_{6}\right) u_{5}=\left(-u_{3}\right) u_{5}=-u_{4} \quad \text { while } \quad u_{7}\left(u_{6} u_{5}\right)=u_{7}\left(-u_{1}\right)=u_{4} \text {. }
$$

$$
\left(u_{6} u_{7}\right) u_{5}=u_{3} u_{5}=u_{4} \quad \text { while } \quad u_{6}\left(u_{7} u_{5}\right)=u_{6}\left(-u_{2}\right)=-u_{4}
$$


For (f)

$$
\left(u_{7} u_{5}\right) u_{6}=\left(-u_{2}\right) u_{6}=u_{4} \quad \text { while } \quad u_{7}\left(u_{5} u_{6}\right)=u_{7} u_{1}=-u_{4} .
$$

The six possibilities (a) to (f) are dealt with. It has been shown that if $\{i, j, k\}=\{5,6,7\}$, then $\left(u_{i} u_{j}\right) u_{k}=-u_{i}\left(u_{j} u_{k}\right)$.

What cases 1 to 3 show is that $\left(u_{i} u_{j}\right) u_{k}=-u_{i}\left(u_{j} u_{k}\right)$ for all $i, j$ and $k$ of $\{1, \ldots, 7\}$. It is now easy enough to prove that $V$ (multiplication $e(\mu)$ ) is anti-associative. Indeed, if $v_{1}=\sum_{i=1}^{7} u_{i} a_{i}$, $v_{2}=\sum_{j=1}^{7} u_{j} b_{j}$ and $v_{3}=\sum_{k=1}^{7} u_{k} c_{k}\left(a_{i}, b_{j}\right.$ and $c_{k}$ integers $)$, then the product $\left(\left(v_{1}, v_{2}\right) e(\mu), v_{3}\right) e(\mu)$ is just

$$
\sum_{k=1}^{7}\left(\sum_{i=1}^{7} \sum_{j=1}^{7}\left(u_{i} u_{j}\right) a_{i} b_{j}\right) u_{k} c_{k}=\sum_{i=1}^{7} \sum_{j=1}^{7} \sum_{k=1}^{7}\left(u_{i} u_{j}\right) u_{k} a_{i} b_{j} c_{k},
$$

where the right hand side is

$$
\sum_{i=1}^{7} \sum_{j=1}^{7} \sum_{k=1}^{7}-u_{i}\left(u_{j} u_{k}\right) a_{i} b_{j} c_{k}=-\sum_{i=1}^{7} u_{i}\left(\sum_{j=1}^{7} \sum_{k=1}^{7}\left(u_{j} u_{k}\right) b_{j} c_{k}\right) a_{i} .
$$

Since the right hand side of this equation is $-\left(v_{1},\left(v_{2}, v_{3}\right) e(\mu)\right) e(\mu)$ it follows that, when the multiplication in $V$ is $e(\mu)\left(\left(\left\{u_{1}, \ldots, u_{7}\right\}, \mu\right)\right.$ the given sac $(1, p-1,1)$-decomposition), $V$ is anti-associative. However, the anti-commutative nature of $V$ implies that for $u$ and $v$ in $V,(u, u) e(\mu)=(v, v) e(\mu)=0$ so that

$$
((u, u) e(\mu), v) e(\mu)+((v, v) e(\mu), u) e(\mu)=0
$$

and $V$ is terminal. Since $\left(\left(u_{5}, u_{6}\right) e(\mu), u_{7}\right) e(\mu)=\left(u_{5} u_{6}\right) u_{7} \neq 0, V$ is non-basic (see Theorem 7.3). Theorem 10.1 is proved.

COROLLARY 10.2. If $V_{i}, i=1,2$, are two non-basic terminal rings with additive groups (possibly the same) of the form $\mathcal{V}_{p}(7)$ ( $p$ an odd prime), then $V_{1}$ and $V_{2}$ are isomorphic.

Proof. By Theorem 10.1, there must exist respective sac $(1, p-1,1)$-decompositions $\left(\left\{u_{1}, \ldots, u_{7}\right\}, \mu_{1}\right)$ and $\left(\left\{u_{1}^{\prime}, \ldots, u_{7}^{\prime}\right\}, \mu_{2}\right)$ on $\left(V_{1},+\right)$ and on $\left(V_{2},+\right)$ as in Theorem 10.1. A group isomorphism $\tau$ of $\left(V_{1},+\right)$ onto $\left(V_{2},+\right)$ taking $u_{i}$ to $u_{i}^{\prime}, i=1, \ldots, 7$, is by Proposition 9.3, an isomorphism between $V_{1}$ and $V_{2}$. Corollary 10.2 is proved.

By Corollary 10.2 there is, up to isomorphism, only one non-basic terminal ring on a $\mathcal{V}_{p}(7)(p$ an odd prime). Its rather exceptional nature makes it worthy of special notation. We denote it by $n \mathcal{B}\left(\mathcal{V}_{p}(7)\right)$. Here the symbols $n \mathcal{B}$ are used to indicate it is non-basic.

Taken together theorems Theorem 8.3 and Theorem 10.1 provide real enough insight into finite low odd order terminal rings. More is provided in our final three sections.

\section{CERTAIN BASIC TERMINAL RINGS}

Section eight looked at terminal rings of order $p^{n}$ ( $p$ an odd prime and $n$ in $\{1, \ldots, 6\}$ ). Although these were not classified, they were shown to be basic. At this stage the goal before us is to classify terminal rings of order $p^{n}$ ( $p$ an odd prime and $n$ in $\{1,2,3,4\}$ ). The present section does not achieve this. It does however limit such terminal rings still further.

If $V$ is an abelian group, then the null group on $V$ is a basic terminal ring (here the $U$ of Theorem 7.3 can be taken as $\{0\})$. These are the improper basic terminal rings. All others will be called proper. Quite a number of the terminal rings of order $p^{n}$ ( $p$ an odd prime and $n \in\{1,2,3,4\}$ ) are null groups but proper basic terminal rings can occur.

If $k$ is an integer $\geq 1$ then, as has previously been the case, the cyclic group of order $k$ will be denoted by $C_{k}$. For the terminal rings under investigation minimal ideals turn out to be certain $C_{p}$ subgroups (ie. subgroups isomorphic to a $C_{p}$ ). A property of some minimal ideals of a binary group was briefly touched on at the end of section six. The present need is to establish something definite in the case of the terminal rings we are investigating. 
PROPOSITION 11.1. If $V$ is a terminal ring of order $p^{n}$ ( $p$ an odd prime and $n \geq 1$ an integer), then a subgroup $A$ of $(V,+)$ is a minimal ideal if, and only if, it is a $C_{p}$ subgroup of $(V,+)$ with the property that $A V=\{0\}$.

Proof. If $A$ is a $C_{p}$ subgroup of $(V,+)$ with the property that $A V=\{0\}$ then, as it is a minimal normal $V$-subgroup of $V$, it is a minimal ideal. Suppose $A$ is a minimal ideal of $V$. $V$ has ideals $U_{0}, U_{1}, U_{2}$ and $U_{3}$ with $\{0\}=U_{0} \leq U_{1} \leq U_{2} \leq U_{3}=V$ where $U_{i} V \subseteq U_{i-1}$ for $i=1,2$ and 3 (see Theorem 7.2). Take $j$ in $\{0,1,2\}$ maximal such that $A \cap U_{j}=\{0\} . A \leq U_{j+1}$ and, as a consequence, $A V \subseteq U_{j}$. Thus $A V$ is contained in $A$ and $U_{j}$ and must be $\{0\}$. As this means any subgroup of $(A,+)$ is a $V$-subgroup, the minimality of $A$ forces it to be a $C_{p}$ subgroup. Proposition 11.1 is proved.

Let $V$ be as in Proposition 11.1. That result dealt with minimal ideals. The corollary of the next proposition looks at how certain maximal ideals can effect $V$ 's structure.

PROPOSITION 11.2. Suppose $V$ is an anti-commutative ring of order $p^{n}$ ( $p$ an odd prime and $n \geq 1$ an integer) and $A$ a subgroup of $(V,+)$ such that $A V=\{0\}$. If the additive group $(V,+) / A$ is cyclic, then $V$ is a null group.

Proof. It is not difficult to show that the additive group of $V$ is $A+\langle b\rangle$ where $b$ can be taken as an element of $V \backslash A$. Let $a_{i}+b n_{i}\left(i=1,2, a_{i}\right.$ in $A$ and $n_{i}$ integers $\left.\geq 0\right)$ be elements of $V$. We have

$$
\left(a_{1}+b n_{1}\right)\left(a_{2}+b n_{2}\right)=a_{1} a_{2}+a_{1}\left(b n_{2}\right)+\left(b n_{1}\right) a_{2}+\left(b n_{1}\right)\left(b n_{2}\right)=0+0+0+(b b) n_{1} n_{2} .
$$

The anti-commutativity of $V$ forces $b b$ to be 0 . Thus $\left(a_{1}+b n_{1}\right)\left(a_{2}+b n_{2}\right)=0$ and $V$ is a null group. Proposition 11.2 is proved.

COROLLARY 11.3. If $V$ is a terminal ring of order $p^{n}$ ( $p$ an odd prime and $n \geq 1$ an integer) then, when $V$ has a maximal ideal $A$ with $A V=\{0\}$, it must necessarily be a null group.

Proof. As $V / A$ is a simple terminal ring of order $p^{k}(k \geq 1$ an integer) it is, by Proposition 11.1, of order $p$. Thus, the additive group of $V / A$ is cyclic and the result follows from Proposition 11.2. $\quad \square$

In what follows there will be some need to broaden considerations from very restricted terminal rings to anti-commutative rings with similar restriction. From now on (next two sections as well) $V$ will be an anti-commutative ring of order $p^{n}$ ( $p$ an odd prime and $n \in\{1,2,3,4\}$ ). Although it is the case of $V$ terminal that is of interest our study is facilitated by including anti-commutative rings. The results of the next two sections that do not assume $V$ is terminal are Theorems 12.1, 12.3, 13.2, 13.5, 13.8 and 13.10 .

PROPOSITION 11.4. If $n=1$ or 2 and $V$ is terminal, then $V$ is a null group.

Proof. If $n=1$, then $V$ is a simple terminal ring and by Proposition $11.1, V . V=\{0\}$. If $n=2$, then a minimal ideal $A$ of $V$ is, by Proposition 11.1, maximal in $V$. Since, by Proposition 11.1, $A V=\{0\}$, it follows from Corollary 11.3 that $V$ is null. Proposition 11.4 has been proved.

When $n=3$ the possibility of proper basic $V$ arises.

THEOREM 11.5. If $n=3$ and $V$ is terminal, then $V$ is either a null group or properly basic. In the second case $V$ has a unique minimal ideal $A$, the additive group of $V / A$ is of the form $C_{p} \oplus C_{p}, V V \subseteq A$ and $A V=\{0\}$.

Proof. By Theorem 8.3 it follows that, if $V$ is not null, then it is properly basic. Suppose the second case holds and there exist two distinct minimal ideals $A_{1}$ and $A_{2}$ of $V$. It follows from Proposition 11.1 that $A_{1} \oplus A_{2}$ (=B say) is maximal in $V$ and $B V=\{0\}$. As this is contrary to Corollary $11.3, V$ has a unique minimal ideal $A$. Here Proposition 11.4 implies $V V \subseteq A\left(V / A\right.$ has order $\left.p^{2}\right)$ and, by Proposition 11.1, $A V=\{0\}$. Also, in this situation, the order $p^{2}$ group $(V / A,+)$ cannot be cyclic (see Proposition 11.2) and must be of the form $C_{p} \oplus C_{p}$. The theorem is proved.

The case of $n=4$ is considerably more complicated. Once again we have $V$ is null or properly basic but now deeper analysis reveals more is going on.

THEOREM 11.6. If $n=4$ and $V$ is terminal, then $V$ must be one of the following: 
(i) a null group,

(ii) properly basic and of the form $A_{1} \oplus A_{2}\left(A_{i}, i=1,2\right.$, ideals of $\left.V\right)$, where $A_{1}$ has order $p$ and $A_{2}$ is properly basic of order $p^{3}$,

(iii) properly basic with more than one minimal ideal and $(V,+)$ of the form $C_{p^{2}} \oplus C_{p^{2}}$ or $C_{p^{2}} \oplus C_{p} \oplus C_{p}$

(iv) properly basic with a unique minimal ideal $A$ such that $V V \subseteq A$ and $A V=\{0\}$.

Proof. Let us start by supposing $V$ is not a null group and has more than one minimal ideal. Since (ii) and (iii) are the only possibilities for non-null $V$ with more than one minimal ideal, we need to show our $V$ satisfies (ii) or (iii). Certainly $V$ is properly basic (see Theorem 8.3). If $B_{1}$ and $B_{2}$ are two distinct minimal ideals of $V$, then $B_{1} \oplus B_{2}$ (=B say) is an ideal of $V$ such that $B V=\{0\}$ (see Proposition 11.1). However, $V / B$ is a terminal ring of order $p^{2}$. By Proposition 11.4, it readily follows that $V V \subseteq B$. Another fact is that the additive group of $V / B$ cannot be a $C_{p^{2}}$ because this would imply $V$ is null (see Proposition 11.2). Take $a$ and $b$ in $V \backslash B$ such that the subgroup $\langle a, b, a b\rangle$ of $V$ generated by $a, b$ and $a b$ has the property that $\langle a, b, a b\rangle+B=V$. It is not difficult to see $\langle a, b, a b\rangle\langle a, b, a b\rangle \subseteq\langle a, b, a b\rangle$ and, because $\langle a, b, a b\rangle B=\{0\},\langle a, b, a b\rangle$ is an ideal of $V$. Two cases arise. Either $\langle a, b, a b\rangle\langle V$ or $\langle a, b, a b\rangle=V$. It will be shown that in the first of these (ii) holds and in the second we have (iii).

In the first of the two cases it can be seen that, because $B$ is of the form $C_{p} \oplus C_{p}$, it is necessarily of the form $B_{3} \oplus(B \cap\langle a, b, a b\rangle)$ where $B_{3}$ is a subgroup of $B$. The fact that $\langle a, b, a b\rangle+B=V$ can only mean $\langle a, b, a b\rangle \oplus B_{3}=V . B_{3} \neq\{0\}$ since otherwise $\langle a, b, a b\rangle=V$. Also $B_{3} \neq B$ as $B_{3}=B$ would imply $V$ is a direct sum of two null ideals of order $p^{2}$ (see Proposition 11.4) and is necessarily null. Thus, $B_{3}$ is a $C_{p}$ subgroup of $V$ with the property that $B_{3} V=\{0\}$. It must be an ideal (see Proposition 11.1). Clearly the ideal $\langle a, b, a b\rangle$ (of order $p^{3}$ ) cannot be null and, by Theorem 11.5, is properly basic. Thus (ii) holds.

In the $\langle a, b, a b\rangle=V$ situation, $V$ is additively generated by three elements and cannot be of the form $C_{p} \oplus C_{p} \oplus C_{p} \oplus C_{p}$. If it is ruled out that $(V,+)$ is of the form $C_{p^{3}} \oplus C_{p}$ (form $C_{p^{4}}$ ), then only the $C_{p^{2}} \oplus C_{p^{2}}$ and $C_{p^{2}} \oplus C_{p} \oplus C_{p}$ possibilities remain. $C_{p^{3}} \oplus C_{p}\left(C_{p^{4}}\right)$ can indeed be ruled out since such a group has exponent $p^{3}$ (exponent $p^{4}$ ) while $V / B$ and $B$ are $C_{p} \oplus C_{p}$ 's (of exponent $p$ ) so that $V$ has exponent $p$ or $p^{2}$. It has been shown that a non-null $V$ with more than one minimal ideal satisfies (ii) or (iii).

If it is shown that a non-null $V$ with a unique minimal ideal $A$ satisfies (iv), then the theorem will be proved. This is because, by Theorem 8.3, $V$ is basic. Certainly $A V=\{0\}$ (see Proposition 11.1) and it is only required that $V / A$ be shown to be a null group. To prove this suppose the contrary. In this case there exists $c+A$ and $d+A(c$ and $d$ in $V)$ with $(c+A)(d+A) \neq A$. Here, $(c+A)(d+A)$ is a non-zero element of the unique minimal ideal of $V / A$ (see Theorem 11.5). Let this ideal be $A^{\prime} / A$ where $A^{\prime}>A$ is an ideal of $V$. As $(c+A)(d+A)=c d+A, c d$ is in $A^{\prime} \backslash A$. (cd) $P_{0}(V)$ is the ideal of $V$ generated by $c d$ and cannot be such that $A \cap\left((c d) P_{0}(V)\right)=\{0\}$ (this would imply $V$ had at least two minimal ideals). A consequence is that $A^{\prime}=(c d) P_{0}(V)$. The next step in obtaining the required contradiction is to show that the subgroup $\langle c, d, c d\rangle$ of $V$ is such that $\langle c, d, c d\rangle+A=V$.

To show this note that $c d+A$ must additively generate the unique ideal $A^{\prime} / A$ (a $C_{p}$ subgroup of $V / A$ ) of $V / A$. Also, neither $c+A$ nor $d+A$ can be in $A^{\prime} / A$ (this would imply $\left.(c+A)(d+A)=A\right)$. Along with this the subgroup $\left\langle c+A+A^{\prime} / A, d+A+A^{\prime} / A\right\rangle$ (of order $p$ or $p^{2}$ ) of $(V / A) /\left(A^{\prime} / A\right)$ has order $p^{2}$ because otherwise $c+A+A^{\prime} / A=d n+A+A^{\prime} / A\left(n \geq 1\right.$ an integer) so that $c=d n+a^{\prime}\left(a^{\prime} \in A^{\prime}\right)$. As this would imply $c d$ were to be in $A,(V / A) /\left(A^{\prime} / A\right)$ must coincide with $\left\langle c+A+A^{\prime} / A, d+A+A^{\prime} / A\right\rangle$. It has been shown $(V / A,+)$ is additively generated by $c+A, d+A$ and $c d+A$. Thus, $\langle c, d, c d\rangle+A=V$.

It is not difficult to see from the terminal nature of $V$ that $\langle c, d, c d\rangle\langle c, d, c d\rangle \subseteq\langle c, d, c d\rangle$ and, as $\langle c, d, c d\rangle A=\{0\},\langle c, d, c d\rangle$ is an ideal of $V$. A has order $p$ so that in the case of $\langle c, d, c d\rangle<V$, $\langle c, d, c d\rangle \cap A=\{0\}$ and a minimal ideal of $V$ distinct from $A$ can be found in $\langle c, d, c d\rangle$. This is contrary to the uniqueness of $A$ and the conclusion that $\langle c, d, c d\rangle=V$ has been reached.

The next step in obtaining a contradiction to the assumption that $V / A$ is not a null group is to prove $c d$ has order $p^{2}$. This is easy enough to accomplish. The fact that $V$ is basic and $=\langle c, d, c d\rangle$ 
implies $\langle c d\rangle V=\{0\}$ (see Theorem 7.3). This in turn implies $\langle c d\rangle$ is the ideal $(c d) P_{0}(V)$ of $V$ generated by $c d$ (ie. $A^{\prime}$ ). $A^{\prime}$ was of order $p^{2}$ so that, as required, $|c d|=p^{2}$.

Assuming $V / A$ is not a null group has lead to the conclusion that $c d$ has order $p^{2}$. Disproving this possibility will complete the proof. The $V$ with elements of order $p^{2}$ are additively of the form $C_{p^{2}} \oplus C_{p^{2}}, C_{p^{2}} \oplus C_{p} \oplus C_{p}, C_{p^{3}} \oplus C_{p}$ and $C_{p^{4}}$. In the case of $C_{p^{4}},(V,+)$ is a cyclic group $\langle u\rangle(u \in V)$. As $\langle u\rangle\langle u\rangle=\{0\}, V$ is a null group contrary to the fact that $c d$ is non-zero. In the case of $C_{p^{2}} \oplus C_{p} \oplus C_{p}$ and $C_{p^{3}} \oplus C_{p},(V,+)$ is of the form $C_{r} \oplus D\left(r=p^{2}\right.$ or $p^{3}$ and $D$ a abelian group of exponent $\left.p\right)$. A product of two elements of $V$ is a sum of the product of two elements where at least one is from $D$. A product of two elements with one from $D$ is 0 or of additive order $p$. Thus, the product of two elements is either 0 or of order $p$, contradicting $c d$ being of order $p^{2}$. When $(V,+)$ is of the form $\left\langle u_{1}\right\rangle \oplus\left\langle u_{2}\right\rangle,\left(u_{i}, i=1,2\right.$, elements of $V$ of order $\left.p^{2}\right)$ we have a product of two elements is of the form $\left(u_{1} u_{2}\right) n$ ( $n$ an integer). In this case, if it is shown that $u_{1} u_{2}$ is 0 or of order $p$, then the desired contradiction will be obtained. If $u_{1} u_{2}$ did not have such an order, then $u_{1} u_{2}=u_{1} n_{1}+u_{2} n_{2}$, where $n_{i}$ are integers $\geq 1$ with at least one of them being coprime to $p$. We may assume $n_{1}$ is coprime to $p$ and consider $\left(u_{1} u_{2}\right) u_{2}$. Here $\left(u_{1} u_{2}\right) u_{2}=\left(u_{1} u_{2}\right) n_{1}$ (clearly non-zero) contrary to $\left(u_{1} u_{2}\right) u_{2}=-u_{1}\left(u_{2} u_{2}\right)$ being $0 . c d$ of order $p^{2}$ cannot exist and Theorem 11.6 is proved.

\section{WHEN $N=1,2$ OR 3}

It has been assumed that $V$ is a anti-commutative ring of order $p^{n}$ ( $p$ an odd prime and $n$ in $\{1,2,3,4\}$ ). This section will classify those $V$ which are terminal and have $n=1,2$ or 3 . For $n=1$, there is up to isomorphism, one such $V$. It is the null group on a $C_{p}$ (see Proposition 11.4). Also Proposition 11.4 tells us that for possible $V$ when $n=2$ there is, up to isomorphism, precisely two $V$. These are the null groups on $C_{p^{2}}$ and $C_{p} \oplus C_{p}$. The $n=3$ case is somewhat more complicated.

For $n=3$ there is the null groups on our terminal $V$. Certainly these are determined by $V$ 's additive structure. Thus, as $(V,+)$ can only be $C_{p^{3}}, C_{p^{2}} \oplus C_{p}$ or $C_{p} \oplus C_{p} \oplus C_{p}$, three different $V$ arise. By Theorem 11.5, the non-null $V$ are properly basic with a unique minimal ideal. For $(V,+)=C_{p^{3}}$, $V$ has a unique minimal ideal (the $C_{p}$ subgroup of $V$ ). However here, although $V$ is basic it is not properly basic (ie. $V$ is null). Thus in our classification we are seeking the terminal $V$ with $(V,+)$ of the form $C_{p^{2}} \oplus C_{p}$ or $C_{p} \oplus C_{p} \oplus C_{p}$, which are properly basic (ie. non-null).

Things now return to $V$ being anti-commutative.

THEOREM 12.1. Suppose $n=3$ and $(V,+)$ is of the form $C_{p^{2}} \oplus C_{p} . V$ is a terminal ring and non-null if, and only if, $V$ has a (sac) $)_{2}$-decomposition $(\{u, v\}, \mu)$ where $|u|=p^{2},|v|=p,(u, u) \mu=(v, v) \mu=0$, $(u, v) \mu=-(v, u) \mu=u p$ and multiplication in $V$ being $e(\mu)$.

Proof. First it is proved that, when $V$ is terminal and non-null, $(\{u, v\}, \mu)$ exists. Here the nature of $(V,+)$ implies there exists $u_{1}$ and $v_{1}$ in $V$ with $\left|u_{1}\right|=p^{2},\left|v_{1}\right|=p$ and $(V,+)=\left\langle u_{1}\right\rangle \oplus\left\langle v_{1}\right\rangle$. It is readily seen that the ideal $V p$ of $V$ must be $\left\langle u_{1} p\right\rangle$ (a $C_{p}$ subgroup of $V$ ). The anti-commutative nature of $V$ ensures that a product of two elements is either 0 or of the form $\left(u_{1} v_{1}\right) k(k \geq 1$ an integer). As $V$ is nonnull and $V /\left\langle u_{1} p\right\rangle$ is a terminal ring of order $p^{2}$, we conclude (see Theorem 11.5) that $u_{1} v_{1}=\left(u_{1} p\right) k_{1}$ where $k_{1} \geq 1$ is an integer coprime to $p$. An integer $k_{2} \geq 1$ with $k_{2} k_{1} \equiv 1 \bmod p$ which is coprime to $p$ exists and we now take $u=u_{1} k_{1}$ and $v=v_{1} k_{2}$. It is certainly true that $V=\langle u\rangle \oplus\langle v\rangle$. The fact that $u v=u p$ follows because $u v=\left(u_{1} k_{1}\right)\left(v_{1} k_{2}\right)=\left(u_{1} v_{1}\right)\left(k_{1} k_{2}\right)=\left(u_{1} p\right)\left(k_{1} k_{1} k_{2}\right)$. Here, since $u_{1} p$ has order $p$, the right hand side is just $\left(u_{1} p\right) k_{1}=\left(u_{1} k_{1}\right) p=u p$. It should now be clear that with $\mu$ defined by $(a, b) \mu=a b$ ( $a$ and $b$ in $\{u, v\}),(\{u, v\}, \mu)$ is the required (sac) $)_{2}$-decomposition (by Proposition 9.2 the multiplication in $V$ is $e(\mu))$.

Proving the converse is easy enough. By Proposition 9.1, $V$ under $e(\mu)$ is an anti-commutative ring and the product $\left(a_{1} b_{1}\right) c_{1}\left(a_{1}, b_{1}\right.$ and $c_{1}$ in $\left.V\right)$ is of the form $\left[(u v) k_{3}\right] c_{1}=\left[u\left(p k_{3}\right)\right] c_{1}\left(k_{3} \geq 1\right.$ an integer). Because $c_{1}=u n_{1}+v n_{2}\left(n_{i}, i=1,2\right.$, integers $)$, it follows readily enough that $\left(a_{1} b_{1}\right) c_{1}=u\left(p^{2} k_{3} n_{2}\right)=0$. Theorem 7.3 is applicable and $V$ is terminal. Since $u v \neq 0$ it is non-null. Theorem 12.1 is proved.

COROLLARY 12.2. If $V_{i}, i=1,2$ are two non-null terminal rings with additive groups (possibly the same) of the form $C_{p^{2}} \oplus C_{p}$, then $V_{1}$ and $V_{2}$ are isomorphic. 
Proof. By Theorem 12.1, there exist (sac) $)_{2}$-decompositions $\left(\left\{u_{i}, v_{i}\right\}, \mu_{i}\right)$ on the $\left(V_{i},+\right)$ as in the statement of Theorem 12.1. A group isomorphism $\tau$ of $\left(V_{1},+\right)$ onto $\left(V_{2},+\right)$ taking $u_{1}$ to $u_{2}$ and $v_{1}$ to $v_{2}$ is, by Proposition 9.3, an isomorphism between $V_{1}$ and $V_{2}$. Corollary 12.2 is proved.

We have seen that up to isomorphism, there is a unique non-null terminal ring with an additive group of the form $C_{p^{2}} \oplus C_{p}$. This terminal ring will be denoted by $\mathcal{O}_{p}(2,1)$. The notation here is essentially self explanatory except possibly for the $\mathcal{O}$. $\mathcal{O}$ is used because such a $V$ has only one minimal ideal.

We are now ready to deal with the case where $(V,+)$ is a group of the form $C_{p} \oplus C_{p} \oplus C_{p}$.

THEOREM 12.3. Suppose $n=3$ and $(V,+)$ is of the form $C_{p} \oplus C_{p} \oplus C_{p} . V$ is a non-null terminal ring if, and only if, $V$ has a (sac) $)_{3}$-decomposition $(\{u, v, w\}, \mu)$ where $|u|=|v|=|w|=p,(u, u) \mu=(u, v) \mu=$ $(v, u) \mu=(u, w) \mu=(w, u) \mu=0,(v, w) \mu=-(w, v) \mu=u$ and multiplication in $V$ being $e(\mu)$.

Proof. First it is proved that, when $V$ is terminal and non-null, $(\{u, v, w\}, \mu)$ exists. Here the nature of $V$ and $(V,+)$ implies there exists $u_{1}, v_{1}$ and $w_{1}$ in $V$ with $\left|u_{1}\right|=\left|v_{1}\right|=\left|w_{1}\right|=p,(V,+)=$ $\left\langle u_{1}\right\rangle \oplus\left\langle v_{1}\right\rangle \oplus\left\langle w_{1}\right\rangle$ and $\left\langle u_{1}\right\rangle$ taken as the unique minimal ideal of $V$ (see Proposition 11.1). Since two element products of elements of $V$ with one in $\left\langle u_{1}\right\rangle$ are 0 it follows that $u_{1} u_{1}=u_{1} v_{1}=v_{1} u_{1}=$ $u_{1} w_{1}=w_{1} u_{1}=0$. Because $V$ is non-null and $V V \subseteq\left\langle u_{1}\right\rangle$ it must follow that $v_{1} w_{1}=u_{1} k_{1}$ where $k_{1}$ is an integer $\geq 1$ coprime to $p$. With $(\{u, v, w\}, \mu)$ defined by, $u=u_{1} k_{1}, v=v_{1}, w=w_{1}$ and $(a, b) \mu=a b$ ( $a$ and $b$ in $\{u, v, w\})$ it follows from the definition of sac-decomposition that $(\{u, v, w\}, \mu)$ is a (sac) $)_{3}$-decomposition of $V$. Certainly $(\{u, v, w\}, \mu)$ is a (sac) $)_{3}$-decomposition of the required type (by Proposition 9.2 the multiplication in $V$ has to be $e(\mu)$ ).

Proving the converse is easy enough. By Proposition 9.1, $V$ under $e(\mu)$ is an anti-commutative ring. It can be checked out that $\{u, v, w\} .\{u, v, w\} \subseteq\{u, 0\}$ and $u\{u, v, w\}=\{0\}$. From this it readily follows that $V V \subseteq\langle u\rangle$ and $\langle u\rangle . V=\{0\}$. Thus the product $\left(a_{1} b_{1}\right) c_{1}\left(a_{1}, b_{1}\right.$ and $c_{1}$ in $\left.V\right)$ is contained in $\langle u\rangle . V=\{0\}$ and $\left(a_{1} b_{1}\right) c_{1}=0$. Theorem 7.3 is applicable and $V$ is terminal. Since $v w \neq 0$ it is non-null. Theorem 12.3 is proved.

COROLLARY 12.4. If $V_{i}, i=1,2$ are two non-null terminal rings with additive groups (possibly the same) of the form $C_{p} \oplus C_{p} \oplus C_{p}$, then $V_{1}$ and $V_{2}$ are isomorphic.

Proof. By Theorem 12.3, there exist (sac) $)_{3}$-decompositions $\left(\left\{u_{i}, v_{i}, w_{i}\right\}, \mu_{i}\right)$ on the $\left(V_{i},+\right)$ as in the statement of Theorem 12.3. A group isomorphism $\tau$ of $\left(V_{1},+\right)$ onto $\left(V_{2},+\right)$ taking $u_{1}$ to $u_{2}, v_{1}$ to $v_{2}$ and $w_{1}$ to $w_{2}$ is, by Proposition 9.3, an isomorphism between $V_{1}$ and $V_{2}$. Corollary 12.4 is proved.

We have seen that up to isomorphism, there is a unique non-null terminal ring with an additive group of the form $C_{p} \oplus C_{p} \oplus C_{p}$. This terminal ring will be denoted by $\mathcal{O}_{p}(1,1,1)$. The notation here is rather self explanatory (see above explanation about the notation $\mathcal{O}_{p}(2,1)$ ).

For given $p$ with $n=3$ the $V$ that can arise have been classified. There are the null terminal rings (three in number) and the two non-null ones $\left(\mathcal{O}_{p}(2,1)\right.$ and $\left.\mathcal{O}_{p}(1,1,1)\right)$.

\section{WHEN $N=4$}

In this section classification of the $V$ which are terminal is completed. Dealing with $n=4$ will finalize matters. Before doing this we introduce notation for the null groups $(n=1,2$ and 3 cases also) involved here. For $n=1,(V,+)$ is a $C_{p}$. Here $V$ is a null group and $V$ is also written as $\mathcal{N}_{p}(1)$. For $n=2,(V,+)$ is either $C_{p^{2}}$ or $C_{p} \oplus C_{p}$. Again $V$ is a null group. We respectively denote $V$ by $\mathcal{N}_{p}(2)$ and $\mathcal{N}_{p}(1,1)$. For $n=3,(V,+)$ is either $C_{p^{3}}, C_{p^{2}} \oplus C_{p}$ or $C_{p} \oplus C_{p} \oplus C_{p}$. Only the $(V,+)=C_{p^{3}}$ case forces $V$ to be a null group. We respectively denote the null groups involved by $\mathcal{N}_{p}(3), \mathcal{N}_{p}(2,1)$ and $\mathcal{N}_{p}(1,1,1)$. For $n=4,(V,+)$ is either $C_{p^{4}}, C_{p^{3}} \oplus C_{p}, C_{p^{2}} \oplus C_{p^{2}}, C_{p^{2}} \oplus C_{p} \oplus C_{p}$ or $C_{p} \oplus C_{p} \oplus C_{p} \oplus C_{p}$. Only the $(V,+)=C_{p^{4}}$ case forces $V$ to be a null group. We respectively denote the null groups involved by $\mathcal{N}_{p}(4), \mathcal{N}_{p}(3,1), \mathcal{N}_{p}(2,2), \mathcal{N}_{p}(2,1,1)$ and $\mathcal{N}_{p}(1,1,1,1)$.

Assume our anti-commutative $V$ is terminal. The partial classification of the $n=4$ situation addressed in Theorem 11.6 will supply background for what follows. (i) of Theorem 11.6 provides us with the five null groups just listed. As $\mathcal{O}_{p}(2,1)$ and $\mathcal{O}_{p}(1,1,1)$ are the only non-null $V$ of order 
$p^{3}$, (ii) of Theorem 11.6 provides us with $\mathcal{N}_{p}(1) \oplus \mathcal{O}_{p}(2,1)$ and $\mathcal{N}_{p}(1) \oplus \mathcal{O}_{p}(1,1,1)$. They are distinct terminal rings as the first contains elements of order $p^{2}$ and the second does not. (iii) of Theorem 11.6 supplies us with the possibility of non-null $V$ having more than one minimal ideal and additive group either $C_{p^{2}} \oplus C_{p^{2}}$ or $C_{p^{2}} \oplus C_{p} \oplus C_{p}$. Investigation into this situation begins with the $C_{p^{2}} \oplus C_{p^{2}}$ case. Any such $V$ necessarily has more than one minimal ideal.

PROPOSITION 13.1. Suppose the anti-commutative ring $V$ is terminal. If $(V,+)$ is of the form $\langle u\rangle \oplus\langle v\rangle$ where $u$ and $v$ in $V$ have additive order $p^{2}$, then the sum $\operatorname{soc}(V)$ of all minimal ideals of $V$ is the subgroup $\langle u p\rangle \oplus\langle v p\rangle$ of $(V,+)$.

Proof. A minimal ideal of $V$ is a $C_{p}$ subgroup of $V$ and $\langle u p\rangle \oplus\langle v p\rangle$ contains all such subgroups. Thus $\operatorname{soc}(V) \leq\langle u p\rangle \oplus\langle v p\rangle$. If it is shown $(u p) w=0$ for all $w$ in $V$, then $\langle u p\rangle V=\{0\}$ and $\langle u p\rangle$ is a minimal ideal of $V$. A similar argument will apply to $\langle v p\rangle$ so that $\langle u p\rangle \oplus\langle v p\rangle$ will have been shown to be $\leq \operatorname{soc}(V)$. In order to arrive at a contradiction assume $(u p) w \neq 0$.

The assumption that $(u p) w \neq 0$ implies $u w \neq 0$. Now $w=u n_{1}+v n_{2}\left(n_{i}, i=1,2\right.$, integers $\left.\geq 1\right)$. Thus $u v \neq 0$. However $u v$ is not of order $p^{2}$. This is because if it were then $u v$ would be of the form $u k_{1}+v k_{2}$ where the $k_{i}, i=1,2$, are integers $\geq 1$ with at least one of them $\left(k_{1}\right.$ say) coprime to $p$. As this implies $(u v) v=(u v) k_{1} \neq 0$ while $(u v) v=-u(v v)=0$ we have a contradiction. Thus $u v$ has order $p$ and $(u p) v=(u v) p=0$. However $(u p) w=(u p)\left(u n_{1}\right)+(u p)\left(v n_{2}\right)=((u v) p) n_{2}=0$. This proves Proposition 13.1.

We are now ready to determine those $V$ with $(V,+)$ of the form $C_{p^{2}} \oplus C_{p^{2}}$ which are both terminal and properly basic (ie. by Theorem 11.6, non-null). Proposition 9.3 will supply the fact that, up to isomorphism, there is one such terminal $V$. The added information of Proposition 13.1 will be of use in labeling it.

THEOREM 13.2. Suppose $n=4$ and $(V,+)$ is of the form $C_{p^{2}} \oplus C_{p^{2}} . V$ is a terminal ring and non-null if, and only if, $V$ has a (sac) $)_{2}$-decomposition $(\{u, v\}, \mu)$ where $|u|=p^{2},|v|=p^{2},(u, u) \mu=(v, v) \mu=0$, $(u, v) \mu=-(v, u) \mu=u p$ and multiplication in $V$ being $e(\mu)$.

Proof. First it is proved that, when $V$ is terminal and non-null, $(\{u, v\}, \mu)$ exists. It may be assumed that $V=\left\langle u_{1}\right\rangle \oplus\left\langle v_{1}\right\rangle$ where $u_{1}$ and $v_{1}$ in $V$ are such that $\left|u_{1}\right|=\left|v_{1}\right|=p^{2}$. If $w_{i}, i=1,2$, are in $V$, then the anti-commutative nature of $V$ ensures $w_{1} w_{2}=\left(u_{1} v_{1}\right) n$ where $n$ is an integer $\geq 1$. Since $V$ is non-null, it follows that $u_{1} v_{1} \neq 0$. It will be shown that $u_{1} v_{1}$ has order $p$. Suppose it does not. Here $u_{1} v_{1}=u_{1} n_{1}+v_{1} n_{2}$ where $n_{i}, i=1,2$, are integers $\geq 1$ with at least one of them ( $n_{1}$ say) coprime to $p$. As this implies $\left(u_{1} v_{1}\right) v_{1}=\left(u_{1} n_{1}\right) v_{1}=\left(u_{1} v_{1}\right) n_{1} \neq 0$ while $\left(u_{1} v_{1}\right) v_{1}=-u_{1}\left(v_{1} v_{1}\right)=0$, we conclude $u_{1} v_{1}$ does indeed have order $p$. Three cases arise. These are $u_{1} v_{1}=\left(u_{1} p\right) k_{1}, u_{1} v_{1}=\left(v_{1} p\right) k_{2}$ or $u_{1} v_{1}=\left(u_{1} p\right) m_{1}+\left(v_{1} p\right) m_{2}$ where, in each case, the integers $k_{1}, k_{2}$ and those of $\left\{m_{1}, m_{2}\right\}$ are $\geq 1$ and coprime to $p$.

CASE 1. Here $u_{1} v_{1}=\left(u_{1} p\right) k_{1}=\left(u_{1} k_{1}\right) p$.

An integer $k_{3} \geq 1$ with $k_{3} k_{1} \equiv 1 \bmod p$ which is coprime to $p$ exists and we now take $u=u_{1} k_{1}$ and $v=v_{1} k_{3}$. It is certainly true that $V=\langle u\rangle \oplus\langle v\rangle$. The fact that $u v=u p$ follows because $u v=\left(u_{1} k_{1}\right)\left(v_{1} k_{3}\right)=\left(u_{1} v_{1}\right)\left(k_{1} k_{3}\right)=\left(u_{1} p\right)\left(k_{1} k_{1} k_{3}\right)$. Here, since $u_{1} p$ has order $p$, the right hand side is just $\left(u_{1} p\right) k_{1}=\left(u_{1} k_{1}\right) p=u p$. It should now be clear that with $\mu$ defined by $(a, b) \mu=a b$ ( $a$ and $b$ in $\{u, v\}),(\{u, v\}, \mu)$ is the required (sac) 2 -decomposition (by Proposition 9.2 the multiplication in $V$ is $e(\mu))$.

CASE 2. Here $u_{1} v_{1}=\left(v_{1} p\right) k_{2}=\left(v_{1} k_{2}\right) p$

This follows in a straightforward manner from case 1 . With $v_{1}, u_{1}$ and $k_{2}$ respectively taken as the $u_{1}, v_{1}$ and $k_{1}$ in the above case, the existence of the required (sac) $)_{2}$-decomposition is established.

CASE 3. Here $u_{1} v_{1}=\left(u_{1} p\right) m_{1}+\left(v_{1} p\right) m_{2}$.

An integer $m_{3} \geq 1$ with $m_{3} m_{1} \equiv 1 \bmod p$ which is coprime to $p$ exists and we now take $u=u_{1} m_{1}+v_{1} m_{2}$ and $v=v_{1} m_{3}$. Because $m_{1}$ and $m_{2}$ are coprime to $p, u$ has order $p^{2}$. Also the 
subgroup $\langle u\rangle$ of $V$ intersects $\left\langle v_{1}\right\rangle(=\langle v\rangle)$ in $\{0\}$ (no non-zero element of $\left\langle u_{1} m_{1}+v_{1} m_{2}\right\rangle$ is in $\left\langle v_{1}\right\rangle$ ). With the $u$ and $v$ so defined it follows that $V=\langle u\rangle \oplus\langle v\rangle$. The fact that $u v=u p$ follows because

$$
u v=\left(u_{1} m_{1}+v_{1} m_{2}\right)\left(v_{1} m_{3}\right)=\left(u_{1} v_{1}\right)\left(m_{1} m_{3}\right)=\left[\left(u_{1} p\right) m_{1}+\left(v_{1} p\right) m_{2}\right]\left(m_{1} m_{3}\right) .
$$

Here, since $\left(u_{1} p\right) m_{1}+\left(v_{1} p\right) m_{2}$ has order $p$, the right hand side is just $\left(u_{1} p\right) m_{1}+\left(v_{1} p\right) m_{2}=u p$. It should now be clear that with $\mu$ defined by $(a, b) \mu=a b$ (a and $b$ in $\{u, v\}),(\{u, v\}, \mu)$ is the required (sac) $)_{2}$-decomposition (by Proposition 9.2 the multiplication in $V$ is $e(\mu)$ ).

Proving the converse is easy enough. By Proposition 9.1, $V$ under $e(\mu)$ is an anti-commutative ring and the product $\left(a_{1} b_{1}\right) c_{1}\left(a_{1}, b_{1}\right.$ and $c_{1}$ in $\left.V\right)$ is of the form $\left[(u v) k_{4}\right] c_{1}=\left[u\left(p k_{4}\right)\right] c_{1}\left(k_{4} \geq 1\right.$ an integer). Because $c_{1}=u k_{5}+v k_{6}\left(k_{i}, i=5,6\right.$, integers), it follows readily enough that $\left(a_{1} b_{1}\right) c_{1}=u\left(p^{2} k_{4} k_{6}\right)=0$. Theorem 7.3 is applicable and $V$ is terminal. Since $u v \neq 0$ it is non-null. Theorem 13.2 is proved.

COROLLARY 13.3. If $V_{i}, i=1,2$ are two non-null terminal rings with additive groups (possibly the same) of the form $C_{p^{2}} \oplus C_{p^{2}}$, then $V_{1}$ and $V_{2}$ are isomorphic.

Proof. By Theorem 13.2, there exist (sac) $)_{2}$-decompositions $\left(\left\{u_{i}, v_{i}\right\}, \mu_{i}\right)$ on the $\left(V_{i},+\right)$ as in the statement of Theorem 13.2. A group isomorphism $\tau$ of $\left(V_{1},+\right)$ onto $\left(V_{2},+\right)$ taking $u_{1}$ to $u_{2}$ and $v_{1}$ to $v_{2}$ is, by Proposition 9.3, an isomorphism between $V_{1}$ and $V_{2}$. Corollary 13.3 is proved.

We have seen that up to isomorphism, there is a unique non-null terminal ring with an additive group of the form $C_{p^{2}} \oplus C_{p^{2}}$. This terminal ring will be denoted by $\mathcal{T}_{p}(2,2)$. The notation here is essentially self explanatory except possibly for the $\mathcal{T} . \mathcal{T}$ is used because such a $V$ has at least two minimal ideals (see Proposition 13.1). This terminal ring is distinct from others that have already arisen. The only possibility of any overlap is with the $\mathcal{N}_{p}(1) \oplus \mathcal{O}_{p}(2,1)$ and $\mathcal{N}_{p}(1) \oplus \mathcal{O}_{p}(1,1,1)$. However, in both these cases the additive group has a $C_{p}$ subgroup direct summand. No such subgroup can exist for $C_{p^{2}} \oplus C_{p^{2}}$ so $\mathcal{T}_{p}(2,2)$ is something new.

At this stage things move toward finding an interesting terminal ring with additive group $C_{p^{2}} \oplus C_{p} \oplus C_{p}$. It will turn out to have more than one minimal ideal but not be of type (i) or (ii) of Theorem 11.6. A proposition will be of help in obtaining it.

PROPOSITION 13.4. In the terminal ring $\mathcal{N}_{p}(1) \oplus \mathcal{O}_{p}(2,1)$ an element $v$ of order $p^{2}$ is such that $\langle v\rangle$ is an ideal.

Proof. Clearly, $v=v_{1}+v_{2}$ where $v_{1}$ in $\mathcal{N}_{p}(1)$ is 0 or of order $p$ and $v_{2}$ is in $\mathcal{O}_{p}(2,1)$ and of order $p^{2}$. The additive group of $\mathcal{O}_{p}(2,1)$ is of the form $\left\langle v_{2}\right\rangle \oplus\langle h\rangle$ where $h$ in $\mathcal{O}_{p}(2,1)$ has order $p$. As $\langle v p\rangle=\left\langle v_{2} p\right\rangle=\left[\mathcal{N}_{p}(1) \oplus \mathcal{O}_{p}(2,1)\right] p,\left\langle v_{2} p\right\rangle$ is an ideal of $\mathcal{O}_{p}(2,1)$. Here, $\mathcal{O}_{p}(2,1) /\left\langle v_{2} p\right\rangle$ is a null group (see Proposition 11.4) and so therefore is $\mathcal{N}_{p}(1) \oplus\left[\mathcal{O}_{p}(2,1) /\left\langle v_{2} p\right\rangle\right]$. Thus $\langle v\rangle /\left\langle v_{2} p\right\rangle$ is an ideal of $\mathcal{N}_{p}(1) \oplus\left[\mathcal{O}_{p}(2,1) /\left\langle v_{2} p\right\rangle\right]$ so that $\langle v\rangle$ must be an ideal of $\mathcal{N}_{p}(1) \oplus \mathcal{O}_{p}(2,1)$. Proposition 13.4 is proved.

THEOREM 13.5. Suppose $V$ has additive group $C_{p^{2}} \oplus C_{p} \oplus C_{p} . V$ is a terminal ring not of type (i), (ii) or (iv) of Theorem 11.6 if, and only if, $V$ has a (sac) $)_{3}$-decomposition $(\{u, v, w\}, \mu)$, with $|u|=p^{2}$, $|v|=|w|=p$,

$$
(u, u) \mu=(v, v) \mu=(w, w) \mu=(u, v) \mu=(v, u) \mu=(v, w) \mu=(w, v) \mu=0,
$$

$(u, w) \mu=-(w, u) \mu=v$ and $e(\mu)$ the multiplication in $V$.

Proof. First it is shown that if $V$ is a terminal ring not of type (i), (ii) or (iv) of Theorem 11.6, then $(\{u, v, w\}, \mu)$ exists.

The group $(V,+)$ has elements of order $p^{2}$ and we take $u$ as one of them. It is not difficult to see from the nature of this group that it can be written as $\langle u\rangle \oplus D$ where $D$ is a subgroup of the form $C_{p} \oplus C_{p}$. As $V p=\langle u p\rangle$ is an ideal of $V$ of order $p$ it is a minimal ideal. Having found $u$ the next step is to find $v$ and $w$. We start with finding $w$.

Let $H$ be the sum of all $C_{p}$ subgroups of $V$ and $\operatorname{soc}(V)$ the sum of all ideals. From the $C_{p^{2}} \oplus C_{p} \oplus C_{p}$ nature of $(V,+)$ it follows without any real difficulty that $|H|=p^{3}$. By Proposition 11.1, $\operatorname{soc}(V) \leq H$ and $\operatorname{soc}(V) \cdot V=\{0\}$. If $\operatorname{soc}(V)$ were to be $H$, then it would be maximal in $V$ and Corollary 11.3 
would imply $V$ is null (ie. (i) of Theorem 11.6 holds). Thus $\operatorname{soc}(V)<H$ and $|\operatorname{soc}(V)|=p$ or $p^{2}$. If $|\operatorname{soc}(V)|=p$, then $V$ would have unique minimal ideal, contrary to the fact that (i) and (iv) of Theorem 11.6 do not hold. Thus $|\operatorname{soc}(V)|=p^{2}$. At this stage we take $w$ in $H \backslash \operatorname{soc}(V)$ and, after noting $|w|=p$, proceed to find $v$.

From the fact that $|V / \operatorname{soc}(V)|=p^{2}$ it follows, by Proposition 11.4, that $V / \operatorname{soc}(V)$ is a null group. Thus $(u+\operatorname{soc}(V))(w+\operatorname{soc}(V))=\operatorname{soc}(V)$ and $u w$ is in $\operatorname{soc}(V)$. This element of $V$ is of some interest. It cannot be 0 as $w$ is such that $H w=[\operatorname{soc}(V) \oplus\langle w\rangle] w=0$ (see Proposition 11.1) and if $u w$ were to be 0 then, because $H+\langle u\rangle=V$ we would have $V\langle w\rangle=\{0\}$. Since this would imply the $C_{p}$ subgroup $\langle w\rangle$ of $V$ is a minimal ideal (see Proposition 11.1) contrary to $w$ being in $H \backslash \operatorname{soc}(V)$ we see $u w \neq 0$. Also, as $u w$ is in $\operatorname{soc}(V)$ it has order $p$. The crucial thing here is that $v$ can be taken as $u w$. Furthermore, as $v$ is in $\operatorname{soc}(V),\langle v\rangle V=0, u u=v v=w w=v u=u v=v w=w v=0$ and $u w=-w u=v$. It should be clear from this that if it were to be shown that $V=\langle u\rangle \oplus\langle v\rangle \oplus\langle w\rangle$, then the (sac) 3 -decomposition $(\{u, v, w\}, \mu)$ given by $(a, b) \mu=a b$ ( $a$ and $b$ in $\{u, v, w\}$ ) would be as in the statement of the theorem (here Proposition 9.2 will ensure $e(\mu)$ is the multiplication of $V$ ). Thus the theorem will be proved in the forward direction by showing $(V,+)=\langle u\rangle \oplus\langle v\rangle \oplus\langle w\rangle$.

The required direct sum nature of $(V,+)$ is now established by first proving $(\langle u\rangle+\langle v\rangle) \cap\langle w\rangle=\{0\}$ and then showing $\langle u\rangle \cap\langle v\rangle=\{0\}$. $\langle w\rangle$ is a group of order $p$ so that if $(\langle u\rangle+\langle v\rangle) \cap\langle w\rangle \neq\{0\}$, then $\langle w\rangle \leq\langle u\rangle+\langle v\rangle$. The subgroup of $\langle u\rangle+\langle v\rangle$ of elements of order $p$ can, without any real difficulty, be shown to be $\langle u p\rangle+\langle v\rangle$. Thus $w$ is in $\langle u p\rangle+\langle v\rangle$. The manner in which $v$ was defined ensures $\langle v\rangle \leq \operatorname{soc}(V)$. Also, $\langle u p\rangle(=V p)$ is a minimal ideal of $V$. This is a contradiction as here $w$ (an element of $H \backslash \operatorname{soc}(V))$ has been shown to be in $\operatorname{soc}(V)$. We conclude that $(\langle u\rangle+\langle v\rangle) \cap\langle w\rangle=\{0\}$.

To show $\langle u\rangle \cap\langle v\rangle=\{0\}$ suppose otherwise. As $\langle v\rangle$ is a $C_{p}$ subgroup of $V$ this must mean $v$ is in $\langle u\rangle$. However, $v=u w$ so that $\langle u, w\rangle\langle u, w\rangle \subseteq\langle u, w\rangle$. It it not difficult to see that an element $h$ of $\operatorname{soc}(V)$ not in $\langle u p\rangle$ is such that the sum $\langle h\rangle+\langle u, w\rangle$ is direct. From above $\langle u, w\rangle=\langle u\rangle \oplus\langle w\rangle$ and has order $p^{3}$. Thus $V=\langle u, w\rangle \oplus\langle h\rangle$. The fact that $\langle h\rangle \leq \operatorname{soc}(V)$ means it is an ideal so that, because $\langle u, w\rangle\langle h\rangle=\{0\}$ and $\langle u, w\rangle\langle u, w\rangle \subseteq\langle u, w\rangle$, it can be concluded that $\langle u, w\rangle$ is an ideal. $\langle u, w\rangle$ (of order $p^{3}$ ) is properly basic since $u w \neq 0$. As this contradicts (ii) of Theorem 11.6 not holding, it follows that $\langle u\rangle \cap\langle v\rangle=\{0\}$. Theorem 13.5 has been proved in the forward direction and the only need is to now prove the converse.

Certainly $V$ is an anti-commutative ring under $e(\mu)$. From the definition of $(\{u, v, w\}, \mu)$ we see $\{u, v, w\}\{u, v, w\} \subseteq\{v, 0\}$ and $v\{u, v, w\}=\{0\}$. This implies $V V \subseteq\langle v\rangle$ and $\langle v\rangle V=\{0\}$ so that $\left(a_{1} b_{1}\right) c_{1}\left(a_{1}, b_{1}\right.$ and $c_{1}$ in $\left.V\right)$ is contained in $\langle v\rangle V$ and must be 0 . By Theorem 7.3, $V$ is terminal. Since $u w \neq 0$, (i) of Theorem 11.6 does not hold. Since $u w=v$ (not an element of $\langle u\rangle$ ) $\langle u\rangle$ is not an ideal and, by Proposition 13.4, (ii) of Theorem 11.6 does not hold (previous material implies the only terminal $V$ satisfying (ii) of Theorem 11.6 are $\mathcal{N}_{p}(1) \oplus \mathcal{O}_{p}(2,1)$ and $\mathcal{N}_{p}(1) \oplus \mathcal{O}_{p}(1,1,1)$ where the latter case only has elements of order $p)$. Finally, the definition of $(\{u, v, w\}, \mu)$ implies $u p\{u, v, w\}=v\{u, v, w\}=\{0\}$ so that $\langle u p\rangle V=\langle v\rangle V=\{0\}$ and $\operatorname{soc}(V) \geq\langle u p\rangle \oplus\langle v\rangle$ which must mean $V$ has more than one minimal ideal. Thus (iv) of Theorem 11.6 does not hold and Theorem 13.5 is completely proved.

With straightforward adjustment the proofs of some previous corollaries (eg. Corollary 12.2) supply the verification that:

COROLLARY 13.6. Suppose $V_{i}, i=1,2$, are terminal rings with additive group of the form $C_{p^{2}} \oplus C_{p} \oplus C_{p}$. If the $V_{i}$ do not satisfy (i), (ii) or (iv) of Theorem 11.6, then they are isomorphic.

The unique terminal ring of Corollary 13.6 will be denoted by $\mathcal{T}_{p}(2,1,1)$ (see the definition of $\left.\mathcal{T}_{p}(2,2)\right)$

Terminal rings of type (i), (ii) and (iii) of Theorem 11.6 have been dealt with. We now look at those covered by (iv). The possible additive groups are $C_{p^{4}}, C_{p^{3}} \oplus C_{p}, C_{p^{2}} \oplus C_{p^{2}}, C_{p^{2}} \oplus C_{p} \oplus C_{p}$ and $C_{p} \oplus C_{p} \oplus C_{p} \oplus C_{p}$. Not all these possibilities can occur.

PROPOSITION 13.7. If $n=4$, then $V$ cannot be a terminal ring of type (iv) of Theorem 11.6 when $(V,+)$ has the form $C_{p^{4}}$ or $C_{p^{2}} \oplus C_{p^{2}}$. 
Proof. It is easily seen that when $(V,+)=C_{p^{4}}, V$ must be null. The $(V,+)=C_{p^{2}} \oplus C_{p^{2}}$ case is excluded by Proposition 13.1. Proposition 13.7 is proved.

The study of terminal $V$ with $n=4,(V,+)$ of the form $C_{p^{3}} \oplus C_{p}, C_{p^{2}} \oplus C_{p} \oplus C_{p}$ or $C_{p} \oplus C_{p} \oplus C_{p} \oplus C_{p}$ and of type (iv) of Theorem 11.6 is now addressed. Things start with $C_{p^{3}} \oplus C_{p}$.

THEOREM 13.8. Suppose $n=4$ and $(V,+)$ is of the form $C_{p^{3}} \oplus C_{p} . V$ is a terminal ring and satisfies (iv) of Theorem 11.6 if, and only if, $V$ has a (sac) $)_{2}$-decomposition $(\{u, v\}, \mu)$ where $|u|=p^{3},|v|=p$, $(u, u) \mu=(v, v) \mu=0,(u, v) \mu=-(v, u) \mu=u p^{2}$ and multiplication in $V$ being $e(\mu)$.

Proof. Theorem 13.8 is established in a somewhat similar manner to that used for Theorem 12.1. For the sake of clarity full treatment is given.

First it is proved that, when $V$ is terminal and satisfies (iv) of Theorem 11.6, $(\{u, v\}, \mu)$ exists. Here the nature of $(V,+)$ implies there exists $u_{1}$ and $v_{1}$ in $V$ with $\left|u_{1}\right|=p^{3},\left|v_{1}\right|=p$ and $(V,+)=\left\langle u_{1}\right\rangle \oplus\left\langle v_{1}\right\rangle$. It is readily seen that the ideal $V p^{2}$ of $V$ must be $\left\langle u_{1} p^{2}\right\rangle$ (a $C_{p}$ subgroup of $V$ ). Thus $V p^{2}$ must be the $A$ of (iv) of Theorem 11.6. The anti-commutative nature of $V$ ensures that a product of two elements of $V$ is either 0 or of the form $\left(u_{1} v_{1}\right) k(k \geq 1$ an integer). Also, because (iv) of Theorem 11.6 holds, the non-zero case occurs. Again, since (iv) of Theorem 11.6 holds, $V /\left\langle u_{1} p^{2}\right\rangle$ is a null ring and we conclude that $u_{1} v_{1}=\left(u_{1} p^{2}\right) k_{1}$ where $k_{1} \geq 1$ is an integer coprime to $p$. An integer $k_{2} \geq 1$ with $k_{2} k_{1} \equiv 1 \bmod p$ which is coprime to $p$ exists and we now take $u=u_{1} k_{1}$ and $v=v_{1} k_{2}$. It is certainly true that $V=\langle u\rangle \oplus\langle v\rangle$. The fact that $u v=u p^{2}$ follows because $u v=\left(u_{1} k_{1}\right)\left(v_{1} k_{2}\right)=\left(u_{1} v_{1}\right)\left(k_{1} k_{2}\right)=\left(u_{1} p^{2}\right)\left(k_{1} k_{1} k_{2}\right)$. Here, since $u_{1} p^{2}$ has order $p$, the right hand side is just $\left(u_{1} p^{2}\right) k_{1}=\left(u_{1} k_{1}\right) p^{2}=u p^{2}$. It should now be clear that with $\mu$ defined by $(a, b) \mu=a b(a$ and $b$ in $\{u, v\}),(\{u, v\}, \mu)$ is the required (sac) $)_{2}$-decomposition (by Proposition 9.2 the multiplication in $V$ is $e(\mu))$.

Proving the converse is easy enough. Certainly $V$ under $e(\mu)$ is an anti-commutative ring and the product $\left(a_{1} b_{1}\right) c_{1}\left(a_{1}, b_{1}\right.$ and $c_{1}$ in $\left.V\right)$ is of the form $\left[(u v) k_{3}\right] c_{1}=\left[u\left(p^{2} k_{3}\right)\right] c_{1}\left(k_{3} \geq 1\right.$ an integer). Because $c_{1}=u n_{1}+v n_{2}\left(n_{i}, i=1,2\right.$, integers $)$, it follows readily enough that $\left(a_{1} b_{1}\right) c_{1}=u\left(p^{4} k_{3} n_{2}\right)=0$. Theorem 7.3 is applicable and $V$ is terminal. If $A_{1}$ were a minimal ideal of $V$ distinct from the minimal ideal $\left\langle u p^{2}\right\rangle$, then $\left\langle u p^{2}\right\rangle \oplus A_{1}$ would have to be the subgroup of $(V,+)$ consisting of all elements of order $p$. It is readily seen that if this were to be the case $v$ (of order $p$ ) would have to be such that $V v=0$. Since $u v \neq 0$, (iv) of Theorem 11.6 must hold. Theorem 13.8 is proved.

As is easily seen:

COROLLARY 13.9. If $V_{i}, i=1,2$ are two terminal rings as in (iv) of Theorem 11.6 with additive groups of the form $C_{p^{3}} \oplus C_{p}$, then $V_{1}$ and $V_{2}$ are isomorphic.

The natural notation $\mathcal{O}_{p}(3,1)$ is used for the terminal ring of Corollary 13.9 (see explanation for the notation $\left.\mathcal{O}_{p}(2,1)\right)$.

The examination of terminal $V$ with $n=4$ and $V$ satisfying (iv) of Theorem 11.6 continues. All that remains to be considered are the cases of $(V,+)$ being $C_{p^{2}} \oplus C_{p} \oplus C_{p}$ or $C_{p} \oplus C_{p} \oplus C_{p} \oplus C_{p}$. A definition of help in the $(V,+)=C_{p^{2}} \oplus C_{p} \oplus C_{p}$ situation follows.

If $W$ is an abelian group of the form $C_{p^{2}} \oplus C_{p} \oplus C_{p}$, then a $(2,1,1)$-triple of $W$ will be a triple $(a, b, c)$ where $a, b$ and $c$ are in $W,|a|=p^{2},|b|=|c|=p$ and $W=\langle a\rangle \oplus\langle b\rangle \oplus\langle c\rangle$.

THEOREM 13.10. Suppose $V$ has additive group $C_{p^{2}} \oplus C_{p} \oplus C_{p} . V$ is a terminal ring satisfying (iv) of Theorem 11.6 if, and only if, $V$ has a (sac) $)_{3}$-decomposition $(\{u, v, w\}, \mu)$, with $|u|=p^{2},|v|=|w|=p$,

$$
(u, u) \mu=(v, v) \mu=(w, w) \mu=(u, v) \mu=(v, u) \mu=(u, w) \mu=(w, u) \mu=0,
$$

$(v, w) \mu=-(w, v) \mu=u p$ and $e(\mu)$ the multiplication in $V$.

Proof. First it is shown that if $V$ is a terminal ring satisfying (iv) of Theorem 11.6, then $(\{u, v, w\}, \mu)$ exists. A step toward this is to show for any $(2,1,1)$-triple $(a, b, c)$ of $(V,+), b c \neq 0 . V p(=\langle a p\rangle)$ is an ideal of $V$ of order $p$ and therefore the unique minimal ideal. By (iv) of Theorem 11.6 we have $V V \subseteq\langle a p\rangle$ and the map taking $d$ in $\langle b, c\rangle$ to $a d$ is a group homomorphism of $\langle b, c\rangle$ to $\langle a p\rangle$. Because $\langle b, c\rangle$ has order $p^{2}$ and $\langle a p\rangle$ order $p$, it follows that there exists a $g \neq 0$ in $\langle b, c\rangle$ such that $a g=0$. 
This allows us to conclude that $b c \neq 0$ since if $b c=0$, then $g b=g c=0$ and $g\{a, b, c\}=\{0\}$. It is clear enough from this that, $\langle g\rangle(\leq\langle b, c\rangle)$ is a minimal ideal distinct from $\langle a p\rangle$, forcing $b c$ to be $\neq 0$. This argument in fact gives more. It supplies an element $g \neq 0$ of $\langle b, c\rangle$ with $a g=0$.

Using what has just been established, it is shown that there is a $(2,1,1)$-triple $\left(a_{1}, b_{1}, c_{1}\right)$ of $(V,+)$ with $a_{1} c_{1}=0$ and $b_{1} c_{1} \neq 0$. Let $(a, b, c)$ and $g$ be as above. It is not hard to see that if $g$ is in $\langle b\rangle$, then $\left(a_{1}, b_{1}, c_{1}\right)$ can be taken as $(a, c, b)$. When $g$ is in $\langle c\rangle,\left(a_{1}, b_{1}, c_{1}\right)$ can be taken as $(a, b, c)$. Also, it follows without any real difficulty, that when $g \notin\langle b\rangle \cup\langle c\rangle$, we may take $\left(a_{1}, b_{1}, c_{1}\right)$ as $(a, b, g)$. $\left(a_{1}, b_{1}, c_{1}\right)$ has been found (in any $(2,1,1)$-triple $\left(a^{\prime}, b^{\prime}, c^{\prime}\right)$ on $\left.(V,+), b^{\prime} c^{\prime} \neq 0\right)$. It will be used to establish the existence of a yet more specialized $(2,1,1)$-triple of $(V,+)$.

From the $(2,1,1)$-triple $\left(a_{1}, b_{1}, c_{1}\right)$ of $(V,+)$ another $\left(a_{2}, b_{2}, c_{2}\right)$ is now constructed. Like $\left(a_{1}, b_{1}, c_{1}\right)$ it will have the property $a_{2} c_{2}=0$ (the property $b_{2} c_{2} \neq 0$ must always hold) but $a_{2} b_{2}$ will also be 0 . Clearly if $a_{1} b_{1}=0,\left(a_{2}, b_{2}, c_{2}\right)$ can be taken as $\left(a_{1}, b_{1}, c_{1}\right)$. If $a_{1} b_{1} \neq 0$ then, as $V V \subseteq\left\langle a_{1} p\right\rangle$, $a_{1} b_{1}=a_{1} p r_{1}\left(r_{1} \geq 1\right.$ an integer coprime to $\left.p\right)$. Now $c_{1} b_{1} \neq 0$ so that $c_{1} b_{1}=a_{1} p r_{2}\left(r_{2} \geq 1\right.$ an integer coprime to $p$ ). There exists an integer $r_{3} \geq 1$ such that $r_{2} r_{3} \equiv r_{1} \bmod p$ and, with $\left(a_{2}, b_{2}, c_{2}\right)$ taken as $\left(a_{1}-c_{1} r_{3}, b_{1}, c_{1}\right)$, it will be shown $\left(a_{2}, b_{2}, c_{2}\right)$ is the required $(2,1,1)$-triple. Here $c_{1} r_{3}$ has order $p$ and $a_{2} p=\left(a_{1}-c_{1} r_{3}\right) p=a_{1} p$ so that $\left\langle a_{2}\right\rangle$ has order $p^{2}$ and $\left\langle a_{2} p\right\rangle\left(=\left\langle a_{1} p\right\rangle\right)$ is the unique non-zero proper subgroup of $\left\langle a_{2}\right\rangle$. From this it is easy enough to see that $\left\langle b_{2}, c_{2}\right\rangle$ (of exponent $p$ and $\left.=\left\langle b_{1}, c_{1}\right\rangle\right)$ intersects $\left\langle a_{2}\right\rangle$ in $\{0\}$. Therefore, order considerations ensure $(V,+)=\left\langle a_{2}\right\rangle \oplus\left\langle b_{2}\right\rangle \oplus\left\langle c_{2}\right\rangle$ and $\left(a_{2}, b_{2}, c_{2}\right)$ is indeed a $(2,1,1)$-triple of $(V,+)$. Also, $a_{2} c_{2}=\left(a_{1}-c_{1} r_{3}\right) c_{1}=a_{1} c_{1}=0$ while

$$
a_{2} b_{2}=\left(a_{1}-c_{1} r_{3}\right) b_{1}=a_{1} b_{1}-c_{1} b_{1} r_{3}=a_{1} p r_{1}-a_{1} p r_{2} r_{3}=a_{1} p r_{1}-a_{1} p r_{1}=0 .
$$

The $(2,1,1)$-triple $\left(a_{2}, b_{2}, c_{2}\right)$ of $(V,+)$ has been constructed. It now turns out to be a relatively easy matter to make use of it to find the (sac) $)_{2}$-decomposition $(\{u, v, w\}, \mu)$ of $V$.

The $V$ we are dealing with is such that $V p=\left\langle a_{2} p\right\rangle$ and $\left\langle a_{2} p\right\rangle$ is the unique minimal ideal of $V$. As $V V \subseteq\left\langle a_{2} p\right\rangle$, it follows that $b_{2} c_{2}=a_{2} p r(r \geq 1$ an integer coprime to $p)$. Take $r^{\prime} \geq 1$ as an integer with the property that $r r^{\prime} \equiv 1 \bmod p$. We now take $(u, v, w)$ as $\left(a_{2}, b_{2}, c_{2} r^{\prime}\right)$. It is easy enough to see $(u, v, w)$ is a $(2,1,1)$-triple of $(V,+)$ with the property that $u u=v v=w w=u v=v u=u w=w u=0$ and $v w=b_{2} c_{2} r^{\prime}=a_{2} p r r^{\prime}=a_{2} p=u p$ so that $w v=-u p$. Thus with $(\{u, v, w\}, \mu)$ defined by $(x, y) \mu=x y$ ( $x$ and $y$ in $\{u, v, w\})$ we have the required (sac) $)_{3}$-decomposition (by Proposition 9.2, $e(\mu)$ is the multiplication of $V)$. Theorem 13.10 has been proved in the forward direction.

Certainly $V$ is an anti-commutative ring when the multiplication is taken as $e(\mu)$. To prove the converse of Theorem 13.10 note that $\{u, v, w\}\{u, v, w\} \subseteq\{u, 0\}$ and $u\{u, v, w\}=\{0\}$. This means $V V \subseteq\langle u\rangle$ and $\langle u\rangle V=\{0\}$. As this implies $\left(h_{1} h_{2}\right) h_{3}=0\left(h_{i}, i=1,2,3\right.$, in $\left.V\right), V$ is terminal (see Theorem 7.3). It is properly basic since $v w \neq 0$. Furthermore, if $B$ were a minimal ideal of $V$ distinct from $\langle u p\rangle$, then $(\langle u p\rangle \oplus B) \cap\langle v, w\rangle$ would contain an element $z \neq 0$. Here $\langle v, w\rangle$ is either $\langle z, v\rangle$ or $\langle z, w\rangle$ so that (see above) $z v \neq 0$ or $z w \neq 0$. However, since $(\langle u p\rangle \oplus B) . V=\{0\}$, no such $B$ can exist. Thus (iv) of Theorem 11.6 holds and Theorem 13.10 has been proved.

As is easily seen:

COROLLARY 13.11. If $V_{i}, i=1,2$ are two terminal rings as in (iv) of Theorem 11.6 with additive groups of the form $C_{p^{2}} \oplus C_{p} \oplus C_{p}$, then $V_{1}$ and $V_{2}$ are isomorphic.

The natural notation $\mathcal{O}_{p}(2,1,1)$ is used for the terminal ring of Corollary 13.11 (see explanation for the notation $\left.\mathcal{O}_{p}(2,1)\right)$.

The examination of terminal $V$ with $n=4$ and $V$ satisfying (iv) is almost complete. In the case of $(V+)=C_{p} \oplus C_{p} \oplus C_{p}$ and $V$ with a unique minimal ideal, $\mathcal{O}_{p}(1,1,1)$ was the terminal ring we obtained. On the surface it may look as though something similar will take place for $(V,+)=C_{p} \oplus C_{p} \oplus C_{p} \oplus C_{p}$ (ie. there is an $\left.\mathcal{O}_{p}(1,1,1,1)\right)$. There is however no such terminal ring. A definition will be of help in proving this.

Suppose $V$ is a terminal ring with $(V,+)$ of the form $C_{p} \oplus C_{p} \oplus C_{p} \oplus C_{p}$. A quadruple $(a, b, c, d)$ $(a, b, c$ and $d$ in $V)$ will be called a $(1,1,1,1)$-quadruple of $V$ if $|a|=|b|=|c|=|d|=p,(V,+)=$ $\langle a\rangle \oplus\langle b\rangle \oplus\langle c\rangle \oplus\langle d\rangle$ and $\langle a\rangle$ is a minimal ideal of $V$. 
THEOREM 13.12. There is no terminal $V$ with a unique minimal ideal and $(V,+)$ of the form $C_{p} \oplus C_{p} \oplus$ $C_{p} \oplus C_{p}$.

Proof. Suppose the theorem is false and our $V$ has a unique minimal ideal with $(V,+)$ being of the form $C_{p} \oplus C_{p} \oplus C_{p} \oplus C_{p}$. It is not difficult to see that the null group on $C_{p^{4}}$ is the only terminal ring with a unique minimal ideal satisfying (i), (ii) or (iii) of Theorem 11.6. Thus (iv) of Theorem 11.6 holds and if $A$ is the unique minimal ideal of $V$, then $V V \subseteq A$. $A$ has order $p$ so that with $a$ in $A^{*}$, $\langle a\rangle=A$. The nature of $(V,+)$ ensures there is a $(1,1,1,1)$-quadruple $(a, b, c, d)$ of $V$. The first step toward obtaining a contradiction is to use $(a, b, c, d)$ to provide a $(1,1,1,1)$-quadruple $\left(a_{1}, b_{1}, c_{1}, d_{1}\right)$ of $V$ with $b_{1} c_{1}=0$. As $V V \subseteq\langle a\rangle$, there exists a group homomorphism mapping $h$ of $\langle c, d\rangle$ to $b h$ of $\langle a\rangle$. Now $|\langle c, d\rangle|=p^{2}$ and $|\langle a\rangle|=p$ so that it is possible to find non-zero $g$ in $\langle c, d\rangle$ with $b g=0$. If $g$ is in $\langle c\rangle$ we take $\left(a_{1}, b_{1}, c_{1}, d_{1}\right)$ as $(a, b, c, d)$. When $g$ is in $\langle d\rangle,\left(a_{1}, b_{1}, c_{1}, d_{1}\right)$ is taken as $(a, b, d, c)$. It is not hard to see that for $g$ not in $\langle c\rangle \cup\langle d\rangle,\left(a_{1}, b_{1}, c_{1}, d_{1}\right)$ can be taken as $(a, b, g, d)$.

Assuming the existence of our $V$ there cannot exist a $(1,1,1,1)$-quadruple $\left(a_{2}, b_{2}, c_{2}, d_{2}\right)$ of $V$ with $b_{2} c_{2}=d_{2} c_{2}=0$. This is because in this case $\left\{a_{2}, c_{2}\right\} c_{2}=\{0\},\left\{a_{2}, b_{2}, c_{2}, d_{2}\right\} c_{2}=\{0\}, V .\left\langle c_{2}\right\rangle=\{0\}$ and the subgroup $\left\langle c_{2}\right\rangle$ of order $p$ (disjoint from $\left\langle a_{2}\right\rangle$ ) is a minimal ideal (see Proposition 11.1). $\left(a_{1}, b_{1}, c_{1}, d_{1}\right)$ will be used to obtain just such a $(1,1,1,1)$-quadruple $\left(a_{2}, b_{2}, c_{2}, d_{2}\right)$ of $V$.

In constructing $\left(a_{2}, b_{2}, c_{2}, d_{2}\right)$ things start by noting that if $d_{1} c_{1}=0$, then $\left(a_{2}, b_{2}, c_{2}, d_{2}\right)$ can be taken as $\left(a_{1}, b_{1}, c_{1}, d_{1}\right)$ while if $d_{1} b_{1}$ were to be 0 then because $c_{1} b_{1}=-b_{1} c_{1}=0,\left(a_{2}, b_{2}, c_{2}, d_{2}\right)$ can be taken as $\left(a_{1}, c_{1}, b_{1}, d_{1}\right)$. Thus, it can be assumed that $d_{1} c_{1} \neq 0$ and $d_{1} b_{1} \neq 0$. Here we have $d_{1} c_{1}=a_{1} r_{1}$ and $d_{1} b_{1}=a_{1} r_{2}$ where $r_{i}, i=1,2$, are integers $\geq 1$ and coprime to $p$. Let $r_{3}$ be an integer $\geq 1$ such that $r_{2} r_{3} \equiv r_{1} \bmod p$. Now take $\left(a_{2}, b_{2}, c_{2}, d_{2}\right)$ as $\left(a_{1}, b_{1}, c_{1}-b_{1} r_{3}, d_{1}\right)$. It is not hard to see $\left(a_{2}, b_{2}, c_{2}, d_{2}\right)$ is again a $(1,1,1,1)$-quadruple of $V$. It is certainly true that $b_{2} c_{2}=b_{1}\left(c_{1}-b_{1} r_{3}\right)=0$ and that

$$
d_{2} c_{2}=d_{1}\left(c_{1}-b_{1} r_{3}\right)=d_{1} c_{1}-d_{1} b_{1} r_{3}=a_{1} r_{1}-a_{1} r_{2} r_{3}=a_{1} r_{1}-a_{1} r_{1}=0 .
$$

$\left(a_{2}, b_{2}, c_{2}, d_{2}\right)$ has been constructed and Theorem 13.12 is proved.

The categories (i), (ii) (iii) and (iv) of Theorem 11.6 cover terminal $V$ with $n=4$. The only overlap between them occurs with (ii) and (iii). Those of (ii) or (iii) are $\mathcal{N}_{p}(1) \oplus \mathcal{O}_{p}(2,1), \mathcal{N}_{p}(1) \oplus \mathcal{O}_{p}(1,1,1)$, $\mathcal{T}_{p}(2,2)$ and $\mathcal{T}_{p}(2,1,1)$ (see Corollary 13.3, Proposition 13.1 and Corollary 13.6). Null $V$ with $n=4$ have been discussed and terminal $V$ satisfying (iv) of Theorem 11.6 are $\mathcal{O}_{p}(3,1)$ and $\mathcal{O}_{p}(2,1,1)$ (see Corollary 13.9, 13.11 and Theorem 13.12). For given $p$ the classification of terminal $V$ with $n=4$ is as follows:

THEOREM 13.13. For $n=4$ there are, up to isomorphism, eleven different terminal $V$. They are, $\mathcal{N}_{p}(4)$, $\mathcal{N}_{p}(3,1), \mathcal{N}_{p}(2,2), \mathcal{N}_{p}(2,1,1), \mathcal{N}_{p}(1,1,1,1), \mathcal{N}_{p}(1) \oplus \mathcal{O}_{p}(2,1), \mathcal{N}_{p}(1) \oplus \mathcal{O}_{p}(1,1,1), \mathcal{T}_{p}(2,2), \mathcal{T}_{p}(2,1,1)$, $\mathcal{O}_{p}(3,1)$ and $\mathcal{O}_{p}(2,1,1)$.

Theorem 13.13 is the final result of this paper. Some of the pertinent aspects of terminal rings have been covered. As has been seen these non-associative rings arise from studying the nearring of zero fixing polynomial maps on a binary group. Binary groups are much more general structures. A start on investigating them has been made by me in the 137 page manuscript [6]. Regarding all this it is most certainly appropriate to acknowledge support I have received. The Department of Algebra at Johannes Kepler Universtät (Linz, Austria) graciously hosted me and Gary Peterson for a five week period starting from the end of April 2019. This visit was partially supported by the Austrian Science Fund (FWF) P29931. While there I gave talks relating to structures covered here. Indeed, this paper is a result of the encouragement Erhard Aichinger supplied. I am also very grateful to Gary Peterson for reading through a draft copy and helping with referencing. The support received has been very much an integral part of what has preceded.

\section{REFERENCES}

[1] E. Aichinger. Congruence lattices forcing nilpotency. J. Algebra Appl., 17(2):1850033, 2018.

[2] P. Higgins. Groups with multiple operations. Proc. Lon. Math. Soc., 6(3):366-416, 1956.

[3] H. Neumann. Varieties of groups and their associated nearrings. Math. Z., 65:36-69, 1956. 
[4] G. Pitz. Nearrings. Revised Edition. North-Holland. Amsterdam (1983)

[5] R. SchAfER. An introduction to non-associative algebras. Academic Press, New York (1966)

[6] S. ScotT. Binary groups. 1-137, 2019. Manuscript.

[7] S. Scotт. $N$-solubility and $N$-nilpotency in tame $N$-groups. Alg. Coll. 5, 4 (1998), 425-448.

[8] S. Scotr. Tame nearrings and N-groups. Proc. Edin. Math. Soc. 23, (1980), 275-296.

[9] S. Sсотт. The structure of $\Omega$-groups. Nearrings, nearfields and K-loops, Kluever Acad. Pub., Netherlands, 1997, 47-137.

[10] S. Sсотт. The Z-constrained conjecture. Nearrings and Nearfields. Hamburg, 2003, 69-168. Springer, Dordrecht, 2005.

Open Access statement. This is an open-access article distributed under the terms of the Creative Commons AttributionNonCommercial 4.0 International License (https://creativecommons.org/licenses/by-nc/4.0/), which permits unrestricted use, distribution, and reproduction in any medium for non-commercial purposes, provided the original author and source are credited, a link to the CC License is provided, and changes - if any - are indicated. 